\title{
Nonuniform in Time Input-to-State Stability and the Small-Gain Theorem
}

\author{
Iasson Karafyllis and J. Tsinias
}

\begin{abstract}
For time-varying control systems various equivalent characterizations of the nonuniform in time input-to-state stability (ISS) property are established. These characterizations enable us to derive sufficient conditions for nonuniform in time ISS concerning composite time-varying systems. Our main result generalizes the well-known small-gain theorem due to Jiang-Teel-Praly for autonomous systems under the presence of uniform in time ISS.
\end{abstract}

Index Terms-Lyapunov functions, nonuniform in time input-tostate stability (ISS), small-gain theorem, time-varying systems.

\section{INTRODUCTION}

$\mathbf{T}$ HIS paper constitutes a continuation of authors' work on the concepts of robust global asymptotic stability (RGAS) and input-to-state stability (ISS), nonuniform with respect to initial values of time, and their applicability to stability and feedback stabilization of nonlinear control systems (see [10]-[13], [30]-[32]). We consider nonlinear time-varying systems of the form

$$
\dot{x}=f(t, x, u), x \in \Re^{n}, u \in \Re^{m}, t \in \Re^{+}:=[0,+\infty)
$$

where its dynamics $f: \Re^{+} \times \Re^{n} \times \Re^{m} \rightarrow \Re^{n}$ are continuous with $f(\cdot, 0,0)=0$, namely, $0 \in \Re^{n}$ is an equilibrium, and we assume that $f$ is locally Lipschitz with respect to $(x, u)$, in the sense that for every bounded interval $I \subset \Re^{+}$ and compact subset $S$ of $\Re^{n} \times \Re^{m}$, there is a constant $L \geq 0$ with $|f(t, x, u)-f(t, y, v)| \leq L|(x-y, u-v)|$ for every $t \in$ $I,(x, u ; y, v) \in S \times S$.

Our main purpose is to establish various equivalent characterizations of nonuniform in time ISS and to derive sufficient conditions for the validity of nonuniform in time ISS for composite time-varying systems. The main result of the present paper constitutes a generalization of the well-known Small-Gain Theorem due to Jiang-Teel-Praly [7] for autonomous systems.

\section{A. Motivations}

The notions of nonuniform in time RGAS and nonuniform in time ISS are motivated by the problem of feedback stabilization for a wide class of nonlinear systems, that, although fail to be stabilized at a specific equilibrium by continuous static time-invariant feedback law, a smooth $\left(C^{\infty}\right)$

Manuscript received July 11, 2002; revised April 21, 2003, May 20, 2003, and October 13, 2003. Recommended by Associate Editor K. Gu.

I. Karafyllis is with the Department of Economics. the University of Athens, 10559 Athens, Greece.

J. Tsinias is with the Department of Mathematics, National Technical University of Athens, Athens 15780, Greece (e-mail: jtsin@central.ntua.gr).

Digital Object Identifier 10.1109/TAC.2003.822861 time-varying feedback can be found in such a way that the equilibrium for the resulting closed-loop time-varying system is nonuniformly in time RGAS. Problems, like feedback stabilization for autonomous systems with uncertainties, as well as stabilization at a reference trajectory are reduced to the study of nonuniform in time RGAS and ISS at a specific equilibrium of a system, whose dynamics are time-dependent being in general unbounded with respect to time. We mention here the authors' works [10]-[13], [30]-[32], where stabilization is exhibited for a wide class of systems - including those having triangular structure-by means of smooth time-varying feedback in such a way that the origin of the resulting closed-loop system is nonuniformly in time RGAS. We give below some interesting examples of autonomous systems for which a continuous time-invariant feedback stabilizer, exhibiting uniform in time asymptotic stabilization, does not exist. The most typical situation of such systems is the case of nonholonomic systems (see, for instance, [4]) and here, let us consider the simplest case of the nonholonomic integrator in chained form with input $\left(u_{1}, u_{2}\right) \in \Re^{2}$

$$
\begin{aligned}
\dot{z} & =u_{1} \\
\dot{x}_{i} & =u_{1} x_{i+1}, \quad i=1, \ldots, n-1 \\
\dot{x}_{n} & =u_{2}
\end{aligned}
$$

for which a $C^{0}$ (static or dynamic) time-invariant feedback exhibiting uniform in time asymptotic stabilization at the origin does not exist. We establish in [10] that there exists a smooth time-varying feedback in such a way that $0 \in \Re^{n+1}$ of the corresponding closed-loop system is nonuniformly in time GAS and further its solution is tending to zero as $t \rightarrow+\infty$ with an exponential rate of convergence. We also mention a couple of interesting engineering applications, both taken from [16]. The first is the problem of controlling a mobile robot moving on an uneven surface, which is described as follows:

$$
\begin{aligned}
\ddot{y}_{1}=u_{1}, \ddot{y}_{2}=-\theta_{1}(t) & y_{2}+\theta_{2}(t) y_{3} \\
& -v_{2}\left(y_{1}\right) y_{2}-v_{3}\left(y_{1}\right) y_{3}+u_{2}, \dot{y}_{3}=\dot{y}_{1} y_{2}
\end{aligned}
$$

where $v_{1}, v_{2}$ are $C^{\infty}$ functions and $\theta_{1}, \theta_{2}$ are unknown time-varying bounded parameters. The second example is the problem of controlling the Cartesian position and orientation of a surface vessel with two independent propellers

$$
\ddot{y}_{1}=u_{1}, \ddot{y}_{2}=u_{2}, \ddot{y}_{3}=\left(u_{1}+c \dot{y}_{1}\right) y_{2}-c \dot{y}_{3}
$$

where $c$ is a constant. The systems above do not satisfy Brockett's necessary condition, hence, there are not $C^{0}$ (static or dynamic) time-invariant feedback laws exhibiting uniform in 
time asymptotic stabilization at the origin. Using a constructive strategy, it is established in [10] that both systems can be stabilized nonuniformly with respect to time by means of smooth time-varying feedback laws and further the solutions of the corresponding closed-loop systems are tending to zero as $t \rightarrow+\infty$ with an exponential rate of convergence. More results are derived in [10] and [12], under the presence of nonuniform in time RGAS. Particularly, necessary and sufficient conditions for stabilization of linear time-varying systems as well as sufficient conditions for stabilization of uncertain composite autonomous systems with two inputs of the form

$$
\begin{aligned}
\dot{z} & =A z+b u_{1} \\
\dot{x}_{i} & =F_{i}\left(\theta, z, u_{1}, x_{1}, \ldots, x_{i}\right)+G_{i}\left(z, u_{1}\right) x_{i+1}, \quad i=1, \ldots, n \\
u_{2} & :=x_{n+1} \\
x & :=\left(x_{1}, \ldots, x_{n}\right),(z, x, \theta) \in \Re^{m} \times \Re^{n} \times \Re^{k},\left(u_{1}, u_{2}\right) \in \Re^{2}
\end{aligned}
$$

are established. The state feedback tracking control problem is also solved in [10] and [12] for a class of nonholonomic systems in chained form whose dynamics have triangular structure. The corresponding sufficient conditions imposed in the previously mentioned papers are much weaker and simpler than those in earlier works on the same problem.

Important progress toward the notions of nonuniform in time RGAS and nonuniform in time ISS has been obtained in [11] and [32], where Lyapunov characterizations for these notions are established for time-varying systems (1.1) with locally Lipschitz dynamics. These characterizations allowed us to derive necessary and sufficient conditions for global stabilization of affine in the control time-varying systems at a specific equilibrium by means of a smooth time-varying static feedback in such a way that the equilibrium is nonuniformly in time RGAS with respect to the resulting closed-loop system. The corresponding results [11, Th. 5.1 and Prop. 5.2] generalize the well-known "Artstein-Sontag" Theorem concerning the autonomous case. It should be pointed out that smoothness around zero of the time-varying feedback is guaranteed in [11] without assuming the "small control property", which has been imposed in [3], [19], and [27]. It is worthwhile to emphasize here an important consequence of the previously mentioned result for the autonomous case $\dot{x}=f(x)+g(x) u$ where the dynamics $f, g$ are locally Lipschitz with $f(0)=0$ and assume that the system admits a $C^{1}$ time-varying control Lyapunov function (CLF) $V=$ $V(t, x)$, namely, $V$ satisfies $V(t, 0)=0, V(t, x) \geq a(|x|)$ for all $(t, x) \in \Re^{+} \times \Re^{n}$ for certain $a \in K_{\infty}$ and there exist continuous functions $\rho, \phi: \Re^{+} \rightarrow \Re^{+}$with $\lim _{t \rightarrow+\infty} \phi(t)=0$, $\int_{0}^{+\infty} \phi(t) d t<+\infty, \rho(0)=0$ and $\rho(s)>0$ for all $s>0$ such that for any $(t, x) \in \Re^{+} \times \Re^{n}$ for which $\partial V / \partial x(t, x) g(x)=0$ it holds that $\partial V / \partial t(t, x)+\partial V / \partial x(t, x) f(x) \leq-\rho(V(t, x))+$ $\phi(t)$. Then, [11, Cor. 5.3] establishes that for every gain function $\gamma=\gamma(t,|x|): \Re^{+} \times \Re^{+} \rightarrow \Re^{+}$there exists a smooth timevarying feedback law $u=k(t, x)$ with $k(\cdot, 0)=0$, exhibiting nonuniform in time ISS stabilization of system at zero; particularly, the resulting system $\dot{x}=f(x)+g(x)(k(t, x)+v)$, with $v \in \Re^{m}$ as input satisfies the nonuniform in time ISS property with gain $\gamma$. An explicit formula for the feedback stabilizer $k$ in terms of the CLF above is also provided in [11, Prop. 5.2].

\section{B. Organization and Results}

In Section II, we first provide the notions of nonuniform in time RGAS and ISS as given in [11]. It should be pointed out that the concept of nonuniform in time ISS as proposed in [11] extends the ISS property as described in [28] for the autonomous case. In Section III, we provide the nonuniform in time extension of the familiar notion of uniform in time ISS - as originally proposed by Sontag in [20] for autonomous systems (see also versions of this property in [5] and [15]) — and we establish in Proposition 3.1 equivalence between this notion and the concept of ISS as suggested in [28] and [29]. More equivalent characterizations of nonuniform in time ISS are also established in Proposition 3.1 and links between this notion and the concepts of converging-input-converging-state (CICS) and bounded-input-bounded-state (BIBS) are also given in Corollary 3.5. An interesting consequence of Proposition 3.1 is Proposition 3.7 concerning the autonomous case

$$
\dot{x}=f(x, u),(x, u) \in \Re^{n} \times \Re^{m} .
$$

Particularly, we prove that, if (1.2) is forward complete and satisfies the 0-GAS property, namely, $0 \in \Re^{n}$ is GAS with respect to the unforced system $\dot{x}=f(x, 0)$, then (1.2) satisfies the nonuniform in time ISS property. A typical case of systems that satisfy the previous assumptions and do not satisfy the uniform in time ISS property are bilinear systems $\dot{x}=A x+\sum_{i=1}^{m} u_{i} B_{i} x$ with $A$ being a Hurwitz matrix (see [24]). It is also worthwhile to mention here the two-dimensional counter-example provided in [2], for which neither ISS, or its weaker version "integral ISS", are fulfilled, however, is forward complete and satisfies the 0-GAS property. A consequence of Proposition 3.7 is Corollary 3.8, which asserts that system (1.2) satisfies the nonuniform in time ISS property, if and only if, there is a smooth and everywhere strictly positive function $\phi: \Re^{+} \rightarrow(0,+\infty)$ such that

$$
\dot{x}=f(x, \phi(t) v),(x, v) \in \Re^{n} \times \Re^{m}, t \in \Re^{+}
$$

satisfies the uniform in time ISS property. In Section IV, we derive sufficient conditions for nonuniform in time ISS for composite time-varying systems

$$
\begin{aligned}
& \dot{x}=f(t, x, y, u) \\
& \dot{y}=g(t, x, y, u) \\
& x \in \Re^{n}, y \in \Re^{k}, u \in \Re^{m}, t \in \Re^{+}
\end{aligned}
$$

where $f(t, 0,0,0)=0, g(t, 0,0,0)=0$ for all $t \geq 0$ and $f, g$ are $C^{0}$ mappings, being locally Lipschitz with respect to $(x, y, u)$. The corresponding result (Theorem 4.1) is one of the main results of this paper and constitutes an extension of the well-known small-gain theorem due to Jiang-Teel-Praly in [7], and its relative extensions for autonomous systems under the presence of uniform in time ISS (see, for instance, [8], [9], [25], and [26]). For the autonomous case (1.4), namely, when both $f, g$ are independent of $t$, it is known that uniform in time ISS for (1.4a) with $(y, u)$ as input and for (1.4b) with $(x, u)$ as input lead to a simple sufficient condition under which the overall system satisfies the ISS property from the input $u$. For the time-varying case (1.4) we establish in Theorem 4.1 that a 
set of additional conditions concerning (1.4a) and $(1.4 \mathrm{~b})$ guarantees nonuniform in time ISS for the overall system (1.4). For the special case of (1.4) where $g(\cdot)$ is independent of $x$, namely, for the cascade interconnection

$$
\begin{aligned}
& \dot{x}=f(t, x, y, u) \\
& \dot{y}=g(t, y, u)
\end{aligned}
$$

the sufficient conditions of Theorem 4.1 are considerably simplified. Particularly, Corollary 4.3 provides sufficient conditions for nonuniform in time ISS for (1.5) and generalizes a wellknown result from the autonomous case, which asserts that (1.5) satisfies the ISS property from the input $u$, provided that both (1.5a) with $(y, u)$ as input and (1.5b) with $u$ as input satisfy the ISS property. Finally, in Section V we provide some applications and numerical examples of our Small-Gain Theorem 4.1. Among other things, we combine the results of Corollary 3.8 to derive sufficient conditions for the existence of a smooth output time-varying feedback stabilizer for the autonomous case

$$
\begin{aligned}
& \dot{x}=f(x, y) \\
& \dot{y}=g(x, y)+u \\
& x \in \Re^{n}, y \in \Re, u \in \Re
\end{aligned}
$$

where $f, g$, are $C^{1}$ mappings both vanishing at zero $0 \in \Re^{n+1}$ and only the component $y$ of the solution is available. We make the following hypotheses:

* System (1.6a) satisfies the $0-$ GAS property.

* System (1.6a) is forward complete with $y$ as input.

*The matrix $A:=\frac{\partial f}{\partial x}(0,0)$ is Hurwitz.

By applying the results of Proposition 3.7 and Theorem 4.1, we establish in Example 5.1 that, under (1.7a)-(1.7c), there exists a smooth time-varying feedback law $k:=k(t, y)$ with $k(\cdot, 0)=0$ in such a way that the closed-loop system (1.6) with $u=k(t, y)+v$ satisfies the nonuniform in time ISS property from the input $v$. This result generalizes the corresponding result in [7], which states that, if (1.7c) holds and if we strengthen (1.7a), (1.7b) by assuming uniform in time ISS for (1.6a) with $y$ as input, then there exists a smooth time-invariant feedback law $k:=k(y)$ with $k(0)=0$ exhibiting uniform in time ISS from the input $v$ for the resulting closed-loop system with $u=k(y)+v$. We note here that, under certain additional assumptions, our previous result for the case (1.6) can be extended to the partial state global stabilization problem for triangular systems but we do not aim in the present paper to provide such generalizations in terms of our Small-Gain Theorem 4.1. We report instead the recent works [11], [13], and [30] toward global stabilization of such systems, that contain more results on the stability of the composite system, based on backstepping design being, more or less, equivalent to Theorem 4.1-based approach adopted here for the case (1.6). Another application of Theorem 4.1 is the study of nonuniform in time GAS of linear time-varying interconnected systems

$$
\dot{x}=A_{11}(t) x+A_{12}(t) y+B_{1}(t) u
$$

$$
\begin{aligned}
& \dot{y}=A_{21}(t) x+A_{22}(t) y+B_{2}(t) u \\
& x \in \Re^{n}, y \in \Re^{k}, u \in \Re^{m}, t \in \Re^{+}
\end{aligned}
$$

where all elements of matrices above are real continuous functions of time, being in general unbounded on $\Re^{+}$. In Example 5.2 a set of sufficient conditions are provided, which, according to the Small-Gain Theorem 4.1, guarantee that (1.8) satisfies the nonuniform in time ISS property.

Notations: Throughout this paper we adopt the following notations.

- By $M_{D}$ we denote the set of all measurable functions from $\Re^{+}$to $D$, where $D$ is a given compact subset of $\Re^{m}$.

- By $C^{j}(A)\left(C^{j}(A ; \Omega)\right)$, where $j \geq 0$ is a nonnegative integer, we denote the class of functions (taking values in $\Omega$ ) that have continuous derivatives of order $j$ on $A$.

- For $x \in \Re^{n}, x^{T}$ denotes its transpose and $|x|$ its usual Euclidean norm.

- $L_{\text {loc }}^{\infty}(A)$ denotes the set of all measurable functions $u$ : $A \rightarrow \Re^{m}$ that are essentially bounded on any nonempty compact subset of $A$, and $L^{\infty}(A)\left(L^{1}(A)\right)$ denotes the set of all measurable functions $u: A \rightarrow \Re^{m}$ that are essentially bounded (integrable) on $A$.

- By $B[x, r]$ where $x \in \Re^{n}$ and $r \geq 0$, we denote the closed sphere in $\Re^{n}$ of radius $r$, centered at $x \in \Re^{n}$.

- $x(t)=x\left(t, t_{0}, x_{0} ; u\right)$ denotes the solution of (1.1) at time $t \geq t_{0}$ that corresponds to some input $u \in L_{\mathrm{loc}}^{\infty}\left(\left[t_{0},+\infty\right)\right)$, initiated from $x_{0} \in \Re^{n}$ at time $t_{0} \geq 0$.

- $K^{+}$denotes the class of positive $C^{0}$ functions $\phi: \Re^{+} \rightarrow$ $(0,+\infty)$. For the definitions of classes $K, K_{\infty}$, see [14]. By $K L$ we denote the set of all continuous functions $\sigma=$ $\sigma(s, t): \Re^{+} \times \Re^{+} \rightarrow \Re^{+}$with the properties: (i) for each $t \geq 0$ the mapping $\sigma(\cdot, t)$ is of class $K$; (ii) for each $s \geq 0$, the mapping $\sigma(s, \cdot)$ is nonincreasing with $\lim _{t \rightarrow+\infty} \sigma(s, t)=0$.

For the reader's convenience, we have collected below some properties concerning functions of the classes $K^{+}, K_{\infty}, K L$, which are repeatedly used in the rest of this paper.

Fact I [2, Cor. IV.5]: Consider the function $\gamma:\left(\Re^{+}\right)^{2} \rightarrow \Re^{+}$ and assume that for each $s>0$ the mappings $\gamma(\cdot, s)$ and $\gamma(s, \cdot)$ are of class $K$. Then, there exists a function $\sigma \in K_{\infty}$ such that $\gamma(r, s) \leq \sigma(r) \sigma(s)$ for all $(r, s) \in\left(\Re^{+}\right)^{2}$.

Fact II [24, Cor. 10 and Rem. 11]: For each $\gamma \in K_{\infty}$, there exists $\sigma \in K_{\infty}$ such that $\gamma(r s) \leq \sigma(r) \sigma(s)$ for all $(r, s) \in$ $\left(\Re^{+}\right)^{2}$.

Fact III [24, Prop. 7]: Assume that $\sigma \in K L$. Then, there exist functions $a_{1}, a_{2}$ of class $K_{\infty}$ so that $\sigma(s, t) \leq$ $a_{1}^{-1}\left(\exp (-t) a_{2}(s)\right)$ for all $(t, s) \in\left(\Re^{+}\right)^{2}$.

Fact IV [11, Lemma 2.3]: Let $a: \Re^{+} \times \Re^{+} \rightarrow \Re^{+}$such that: i) for each $t \geq 0$ the functions $a(t, \cdot)$ and $a(\cdot, t)$ are nondecreasing; ii) for all $t \geq 0$ it holds $\lim _{s \rightarrow 0^{+}} a(t, s)=a(t, 0)=0$. Then there exist functions $\zeta \in \stackrel{s \rightarrow 0^{+}}{K_{\infty}}$ and $\phi \in K^{+}$such that $a(t, s) \leq \zeta(\phi(t) s)$ for all $(t, s) \in \Re^{+} \times \Re^{+}$.

The proofs of the following facts are found in the Appendix.

Fact $V$ : Let $U \subseteq \Re^{n}$ be a set that satisfies: i) $0 \in U$; ii) for every $r>0$ the set $U \cap B[0, r]$ is compact. Let $k: \Re^{+} \times U \rightarrow \Re$ 
be a $C^{0}$ function with $k(t, 0)=0$ for all $t \geq 0$. Then, there exist $a \in K_{\infty}$ and $\phi \in K^{+}$such that

$$
|k(t, x)| \leq a(\phi(t)|x|), \quad \forall(t, x) \in \Re^{+} \times U
$$

Fact VI: Let $A, B$ be functions of class $K L$. Then, there exists $R \in K L$ such that

$$
\begin{aligned}
\sup _{t_{0} \leq \tau \leq t} A\left(B\left(s, \tau-t_{0}\right), t-\tau\right) & \leq R\left(s, t-t_{0}\right), \\
\forall t & \geq t_{0} \geq 0, s \geq 0 .
\end{aligned}
$$

Fact VII: Let $\sigma$ be a $K L$ function, $\rho$ be of class $K$ and $\phi_{1}, \phi_{2}$ be a pair of class $K^{+}$in such a way that $\lim _{t \rightarrow+\infty} \phi_{2}(t) \rho\left(\phi_{1}(t) \sigma\left(s, t-t_{0}\right)\right)=0$ for all $\left(t_{0}, s\right) \in\left(\Re^{+}\right)^{2}$. Then, there exist $S \in K L$ and $B \in K^{+}$such that

$$
\begin{aligned}
\phi_{2}(t) \rho\left(\phi_{1}(t) \sigma\left(s, t-t_{0}\right)\right) & \leq S\left(B\left(t_{0}\right) s, t-t_{0}\right), \\
\forall t & \geq t_{0} \geq 0, s \geq 0 .
\end{aligned}
$$

\section{REVIEW OF THE NOTIONS OF NONUNIFORM IN TIME RGAS AND ISS}

In this section, we provide the definition of nonuniform in time RGAS and ISS as precisely given in [11]. We consider time-varying systems of the form

$$
\dot{x}(t)=f(t, x(t), d(t)), \quad x \in \Re^{n}, d \in M_{D}, t \in \Re^{+}
$$

where $D \subset \Re^{m}$ is a compact set, $f: \Re^{+} \times \Re^{n} \times D \rightarrow \Re^{n}$ is a $C^{\circ}$ map being locally Lipschitz with respect to $x \in \Re^{n}$ and satisfies $f(t, 0, d)=0$ for all $(t, d) \in \Re^{+} \times D$.

Definition 2.1: We say that zero $0 \in \Re^{n}$ is nonuniformly in time RGAS for (2.1), if for every $t_{0} \geq 0, d \in M_{D}$ and $x_{0} \in \Re^{n}$ the corresponding solution $x(\cdot)$ of (2.1) exists for all $t \geq t_{0}$ and satisfies the following properties.

$P 1$ (Stability): For every $\varepsilon>0, T \geq 0$, it holds that $\sup \left\{|x(t)|: d \in M_{D}, t \geq t_{0},\left|x_{0}\right| \leq \varepsilon, t_{0} \in[0, T]\right\}<+\infty$ and there exists a $\delta:=\delta(\varepsilon, T)>0$ such that $\left|x_{0}\right| \leq \delta, t_{0} \in$ $[0, T] \Rightarrow|x(t)| \leq \varepsilon, \forall t \geq t_{0}, d \in M_{D}$.

$P 2$ (Attractivity): For every $\varepsilon>0, T \geq 0$ and $R \geq 0$, there exists a $\tau:=\tau(\varepsilon, T, R) \geq 0$ such that $\left|x_{0}\right| \leq R, t_{0} \in$ $[0, T] \Rightarrow|x(t)| \leq \varepsilon, \forall t \geq t_{0}+\tau, d \in M_{D}$.

Proposition 2.2 [11]: The origin $0 \in \Re^{n}$ is nonuniformly in time RGAS for (2.1), if and only if there exists a pair of functions $\sigma \in K L, \beta \in K^{+}$such that for every $\left(t_{0}, x_{0}\right) \in \Re^{+} \times \Re^{n}$ and $d \in M_{D}$ the corresponding solution $x(t)$ of (2.1) with initial condition $x\left(t_{0}\right)=x_{0}$ satisfies $|x(t)| \leq \sigma\left(\beta\left(t_{0}\right)\left|x_{0}\right|, t-t_{0}\right)$ for every $t \geq t_{0}$.

Definition 2.3: We say that system (1.1) satisfies the nonuniform in time 0-GAS property, if P1 and P2 of Definition 2.1 are fulfilled for $u \in D:=\{0\}$, namely, $0 \in \Re^{n}$ is nonuniformly in time GAS for the unforced system $\dot{x}=f(t, x, 0)$.

Definition 2.4: Consider system (1.1) and let $\gamma=\gamma(t, s)$ : $\left(\Re^{+}\right)^{2} \rightarrow \Re^{+}$being continuous, locally Lipschitz in $s$ and such that for each fixed $t \geq 0$ the mapping $\gamma(t, \cdot)$ is a positive-definite function. We say that (1.1) satisfies the "weak" nonuniform in time ISS property (wISS) with gain $\gamma$, if each solution $x(t)=x\left(t, t_{0}, x_{0} ; u\right)$ of (1.1) exists for all $t \geq t_{0}$ and satisfies Properties P1 and P2 of Definition 2.1, provided that $u$ is of class $L_{\mathrm{loc}}^{\infty}\left(\left[t_{0},+\infty\right)\right)$ and satisfies $|u(t)| \leq \gamma(t,|x(t)|)$, a.e. for $t \geq t_{0}$. If in addition for each $t \geq 0$, the function $\gamma(t, \cdot)$ is of class $K_{\infty}$, then we say that (1.1) satisfies the nonuniform in time ISS property with gain $\gamma$.

The following proposition summarizes some useful equivalent descriptions of the nonuniform in time wISS property. It constitutes a generalization of the well-known results in [17] and [22].

Proposition 2.5 [11, Prop. 4.3]: Let $\gamma:=\gamma(t, s):\left(\Re^{+}\right)^{2} \rightarrow$ $\Re^{+}$be a continuous function, which is locally Lipschitz in $s$ and such that for each fixed $t \geq 0$ the mapping $\gamma(t, \cdot)$ is a positive-definite function. Then, the following statements are equivalent.

i) System (1.1) satisfies the (nonuniform in time) wISS property with gain $\gamma$.

ii) $0 \in \Re^{n}$ is nonuniformly in time RGAS for the system $\dot{x}=f(t, x, \gamma(t,|x|) d), \quad x \in \Re^{n}, d \in B[0,1] \subset$ $\Re^{m}, t \in \Re^{+}$.

iii) There exist functions $\sigma \in K L$ and $\beta \in K^{+}$such that the following property holds for all $t \geq t_{0}$ :

$$
\begin{aligned}
|u(\tau)| \leq \gamma(\tau,|x(\tau)|) \text { a.e. for } \tau & \in\left[t_{0}, t\right] \Rightarrow \\
\qquad|x(t)| & \leq \sigma\left(\beta\left(t_{0}\right)\left|x_{0}\right|, t-t_{0}\right) .
\end{aligned}
$$

iv) There exist functions $V \in C^{1}\left(\Re^{+} \times \Re^{n} ; \Re^{+}\right), a_{1}$, $a_{2} \in K_{\infty}, \beta \in K^{+}, \rho \in C^{0}\left(\Re^{+} ; \Re^{+}\right)$being positive definite and $\phi \in C^{0}\left(\Re^{+} ; \Re^{+}\right)$with $\lim _{t \rightarrow+\infty} \phi(t)=0$, $\int_{0}^{+\infty} \phi(t) d t<+\infty$ such that the following hold for all $(t, x, u) \in \Re^{+} \times \Re^{n} \times \Re^{m}$ :

$$
\begin{aligned}
a_{1}(|x|) & \leq V(t, x) \leq a_{2}(\beta(t)|x|) ; \\
|u| & \leq\left.\gamma(t,|x|) \Rightarrow \dot{V}(t, x, u)\right|_{(1.1)} \\
& \leq-\rho(V(t, x))+\phi(t) .
\end{aligned}
$$

v) There exist functions $V \in C^{\infty}\left(\Re^{+} \times \Re^{n} ; \Re^{+}\right), a_{1}, a_{2}$ and $\beta$, as in statement iv), such that (2.3) holds for all $(t, x, u) \in \Re^{+} \times \Re^{n} \times \Re^{m}$ with $\rho(s):=s$ and $\phi(\cdot) \equiv 0$.

Remark 2.6: An equivalent description of property iii) is given in [11] as follows.

vi) There exist functions $a_{1}, a_{2}$ of class $K_{\infty}$ and a function $\tilde{\beta}: \Re^{+} \rightarrow \Re^{+}$of class $K^{+}$such that the following property holds for all $t \geq t_{0}$ :

$$
\begin{aligned}
& |u(\tau)| \leq \gamma(\tau,|x(\tau)|) \text { a.e. for } \tau \in\left[t_{0}, t\right] \Rightarrow \\
& a_{1}(|x(t)|) \leq \exp \left(-\left(t-t_{0}\right)\right) \tilde{\beta}\left(t_{0}\right) a_{2}\left(\left|x_{0}\right|\right) .
\end{aligned}
$$

In order to show the equivalence between iii) and vi) let us first assume that (2.4) holds. Using Fact V, there exist functions $a_{3} \in K_{\infty}$ and $\beta \in K^{+}$such that $\tilde{\beta}(t) a_{2}(s) \leq a_{3}(\beta(t) s)$, for all $(t, s) \in\left(\Re^{+}\right)^{2}$. It is then obvious that (2.2) holds with $\sigma(s, t)=$ $a_{1}^{-1}\left(\exp (-t) a_{3}(s)\right)$. Conversely, suppose next that (2.2) holds. We may then recall Fact III, which asserts that there exist functions $a_{1}, \rho$ of class $K_{\infty}$ with $\sigma(s, t) \leq a_{1}^{-1}(\exp (-t) \rho(s))$ for all $(t, s) \in\left(\Re^{+}\right)^{2}$. Also, by Fact II, there exist functions $\tilde{\rho}, a_{2}$ of class $K_{\infty}$ such that $\rho(\beta(t) s) \leq \tilde{\rho}(\beta(t)) a_{2}(s)$ for all $(t, s) \in\left(\Re^{+}\right)^{2}$. The previous discussion, in conjunction with (2.2), implies (2.4) with $\tilde{\beta}(t):=\tilde{\rho}(\beta(t))$. 
We finally provide an interesting result, which is the analogue of [6, Th. 10.4.3].

Proposition 2.7: System (1.1) satisfies the nonuniform in time wISS property from the input $u$, if and only if it satisfies the nonuniform in time 0 -GAS property.

The proof of Proposition 2.7 is an immediate consequence of the following lemma, which is a direct extension of Lemma IV.10 in [2] and constitutes a powerful tool for the analysis in the next section. Its proof is found in the Appendix .

Lemma 2.8: Suppose that the system (1.1) satisfies the nonuniform in time 0-GAS property. Then, for every function $\mu$ of class $K^{+}$, there exist a $C^{\infty}$ map $V: \Re^{+} \times \Re^{n} \rightarrow \Re^{+}$, functions $a_{i}(i=1, \ldots, 4)$ of class $K_{\infty}$, and $p, \kappa$ of class $K^{+}$, such that

$$
\begin{aligned}
& a_{1}(|x|) \leq V(t, x) \leq a_{2}(p(t)|x|), \forall(t, x) \in \Re^{+} \times \Re^{n} \\
& \frac{\partial V}{\partial t}(t, x)+ \frac{\partial V}{\partial x}(t, x) f(t, x, u) \leq-V(t, x) \\
&+ \exp (-2 t) a_{3}\left(\frac{|x|}{\mu(t)}\right) a_{4}(\kappa(t)|u|), \\
& \forall(t, x, u) \in \Re^{+} \times \Re^{n} \times \Re^{m} .
\end{aligned}
$$

The following example illustrates the nature of definitions of nonuniform in time RGAS, wISS and ISS, as well as the result of Proposition 2.7 for the case of linear time-varying systems.

Example 2.9: In [12], we proved that, if the origin of the linear time-varying system

$$
\dot{x}=A(t) x, x \in \Re^{n}, t \in \Re^{+} .
$$

$A \in \Re^{n \times n}$ being a matrix whose elements are continuous functions, is (nonuniformly in time) GAS, then there exists a function $\mu \in K^{+}$with

$$
\int_{0}^{+\infty} \mu(s) d s=+\infty
$$

and a positive-definite $C^{1}$ matrix $P \in \Re^{n \times n}$ such that

$$
\begin{aligned}
P(t) \geq I, \forall t & \geq 0 \\
\dot{P}(t)+P(t) A(t)+A^{T}(t) P(t)+2 \mu(t) P(t) & \leq 0 \\
\text { for all } t & \geq 0
\end{aligned}
$$

where $I$ is the unit matrix of dimensions $n \times n$. Consider now the time-varying control system

$$
\dot{x}=A(t) x+B(t) u, \quad x \in \Re^{n}, u \in \Re^{m}, t \in \Re^{+}
$$

where $A \in \Re^{n \times n}, B \in \Re^{n \times m}$ are continuous matrices in such a way that (2.7) holds. It turns out from Proposition 2.7 that (2.8) satisfies the nonuniform in time wISS property. Moreover, by virtue of $(2.7 \mathrm{~b})$ it holds that

$$
\begin{aligned}
\phi(t)|u| \leq & |x| \Rightarrow x^{T}\left(\dot{P}(t)+P(t) A(t)+A^{T}(t) P(t)\right) x \\
& +x^{T} P(t) B(t) u+u^{T} B^{T}(t) P(t) x \\
\leq & -\mu(t) x^{T} P(t) x, \quad \forall(t, x) \in \Re^{+} \times \Re^{n}
\end{aligned}
$$

where $\phi(t):=2|P(t) B(t)| / \mu(t)$. Evaluating the derivative of $V(t, x):=x^{T} P(t) x$ along the trajectories of (2.8) and using (2.9) we obtain

$$
\begin{aligned}
|u(\tau)| & \leq \gamma(\tau,|x(\tau)|) \text { a.e. for } \tau \in\left[t_{0}, t\right] \Rightarrow \\
|x(t)| & \leq \exp \left(-\frac{1}{2} \int_{0}^{t-t_{0}} \mu(s) d s\right) \beta\left(t_{0}\right)\left|x_{0}\right|
\end{aligned}
$$

where $\gamma(t, s):=s / \phi(t)$ and

$$
\beta(t):=\exp \left(1 / 2 \int_{0}^{t} \mu(s) d s\right)|P(t)|^{1 / 2} .
$$

Clearly, $\gamma(t, \cdot) \in K_{\infty}$ for each $t \geq 0$, thus, by recalling (2.7a), Definition 2.4, and (2.10) it follows that (2.8) satisfies the nonuniform in time ISS property.

\section{ChARACTERIZATIONS OF THE NONUNIFORM IN TIME ISS}

The following proposition provides equivalent characterizations of the nonuniform in time ISS property. As it is mentioned in the Introduction, the proposition below establishes among other things the equivalence between the notion of nonuniform in time ISS as proposed in [11] (see (2.2) of statement iii) of Proposition 2.5) and the Sontag-like version of ISS for the nonuniform in time case (see (3.3) of statement iv) below).

Proposition 3.1: The following statements are equivalent.

i) System (1.1) satisfies the nonuniform in time ISS property.

ii) There exist functions $\rho \in K_{\infty}, \phi, \beta \in K^{+}$and $\sigma \in K L$ such that

$$
\begin{aligned}
\rho(\phi(t)|u(t)|) & \leq|x(t)| \\
\text { a.e. for } t \geq t_{0} \Rightarrow|x(t)| & \leq \sigma\left(\beta\left(t_{0}\right)\left|x_{0}\right|, t-t_{0}\right) .
\end{aligned}
$$

iii) There exist functions $\rho \in K_{\infty}, \phi, \beta \in K^{+}$and $\sigma \in K L$ such that, for every $\left(t_{0}, x_{0}\right) \in \Re^{+} \times \Re^{n}$ and input of class $u \in L_{\mathrm{loc}}^{\infty}\left(\left[t_{0},+\infty\right)\right)$, the corresponding solution $x(t)$ of (1.1) with $x\left(t_{0}\right)=x_{0}$ exists for all $t \geq t_{0}$ and satisfies

$$
\begin{aligned}
& |x(t)| \leq \\
& \max \left\{\sigma\left(\beta\left(t_{0}\right)\left|x_{0}\right|, t-t_{0}\right), \sup _{t_{0} \leq \tau \leq t} \sigma(\beta(\tau) \rho(\phi(\tau)|u(\tau)|), t-\tau)\right\} .
\end{aligned}
$$

iv) There exist functions $\zeta \in K_{\infty}, \beta, \delta \in K^{+}$and $\sigma \in K L$ such that, for every $\left(t_{0}, x_{0}\right) \in \Re^{+} \times \Re^{n}$ and input $u \in$ $L_{\mathrm{loc}}^{\infty}\left(\left[t_{0},+\infty\right)\right)$, the corresponding solution $x(t)$ of $(1.1)$ with $x\left(t_{0}\right)=x_{0}$ exists for all $t \geq t_{0}$ and satisfies

$|x(t)| \leq \max \left\{\sigma\left(\beta\left(t_{0}\right)\left|x_{0}\right|, t-t_{0}\right), \sup _{t_{0} \leq \tau \leq t} \zeta(\delta(\tau)|u(\tau)|)\right\}$.

v) There exist a function $\theta \in K_{\infty}$, being locally Lipschitz on $\Re^{+}$, and a function $\delta \in K^{+}$such that $0 \in \Re^{n}$ is nonuniformly in time RGAS for the system

$$
\dot{x}=f\left(t, x, \frac{\theta(|x|)}{\delta(t)} d\right), d(\cdot) \in M_{B[0,1]} .
$$


vi) System (1.1) satisfies the nonuniform in time 0-GAS Property and there exist functions $\sigma, \zeta \in K_{\infty}$ and $\delta, \mu, \beta \in K^{+}$such that, for every $\left(t_{0}, x_{0}\right) \in \Re^{+} \times \Re^{n}$ and $u=u(t)$ of class $L_{\mathrm{loc}}^{\infty}\left(\left[t_{0},+\infty\right)\right)$, the corresponding solution $x(t)$ of (1.1) with $x\left(t_{0}\right)=x_{0}$ exists for all $t \geq t_{0}$ and satisfies

$$
|x(t)| \leq \mu(t)\left(\sigma\left(\beta\left(t_{0}\right)\left|x_{0}\right|\right)+\sup _{t_{0} \leq \tau \leq t} \zeta(\delta(\tau)|u(\tau)|)\right) .
$$

vii) There exist a $C^{\infty}$ function $V: \Re^{+} \times \Re^{n} \rightarrow \Re^{+}$, functions $a_{1}, a_{2}, a_{3}$ of class $K_{\infty}$ and $\beta, \phi$ of class $K^{+}$such that for all $(t, x, u) \in \Re^{+} \times \Re^{n} \times \Re^{m}$ we have

$$
\begin{aligned}
a_{1}(|x|) \leq V(t, x) & \leq a_{2}(\beta(t)|x|) \\
\frac{\partial V}{\partial t}(t, x)+\frac{\partial V}{\partial x}(t, x) f(t, x, u) & \leq-V(t, x)+a_{3}(\phi(t)|u|) .
\end{aligned}
$$

Remark 3.2: When the functions $\phi$ and $\beta$ are bounded, then (3.1) is equivalent to the uniform in time ISS property, as given in [28] and [29]. Likewise, when $\beta$ and $\delta$ are bounded, then (3.3) is equivalent to ISS property, as originally proposed in [20] by E.D. Sontag. The equivalence between (3.1) and (3.3) generalizes the well known fact that for the autonomous case and, when $\gamma$ is independent of $t$, namely, $\gamma$ is of class $K_{\infty}$, the uniform in time ISS property as given by Sontag, is equivalent to the corresponding characterization given in [28], [29]. Finally, we note that, when $\phi$ and $\beta$ are bounded, then (3.6a), (3.6b) coincide with the Lyapunov characterization given in [22]-[24] for the uniform in time ISS property.

Proof of Proposition 3.1: We prove the implications i) $\Leftrightarrow$ ii) $\Rightarrow$ iii) $\Rightarrow$ iv $) \Rightarrow$ v) $\Rightarrow$ vii) $\Rightarrow$ ii) and iv) $\Leftrightarrow$ vi). i) $\Leftrightarrow$ ii) Suppose that i) holds. According to statement iii) of Proposition 2.5, there exist functions $\sigma \in K L, \beta \in K^{+}$and a continuous function $\gamma(t, s):\left(\Re^{+}\right)^{2} \rightarrow \Re^{+}$, where $\gamma$ is locally Lipschitz in $s, \gamma(t, \cdot) \in K_{\infty}$ for each fixed $t \geq 0$ and in such a way that (2.2) holds. Let $\gamma^{-1}(t, s)$ denote the inverse function of $\gamma(t, s)$ with respect to $s \geq 0$, namely, $\gamma^{-1}(t, s)$ satisfies $\gamma\left(t, \gamma^{-1}(t, s)\right)=\gamma^{-1}(t, \gamma(t, s))=s$, for all $t, s \geq 0$. Clearly, $\gamma^{-1}(t, s)$ is continuous and thus by invoking Fact $\mathrm{V}$ we may find functions $\rho \in K_{\infty}$ and $\phi \in K^{+}$such that $\gamma^{-1}(t, s) \leq$ $\rho(\phi(t) s)$ for all $t, s \geq 0$. The latter inequality in conjunction with (2.2) implies (3.1). Conversely, suppose that (3.1) holds. Let $\tilde{\rho} \in K_{\infty}$ be a function, locally Lipschitz on $\Re^{+}$that satisfies $\tilde{\rho}(s) \leq \rho^{-1}(s)$ for all $s \geq 0$ and let $\gamma(t, s):=\tilde{\rho}(s) / \phi(t)$. It is a direct consequence of (3.1) and the equivalence between i) and ii) of Proposition 2.5, that (1.1) satisfies the nonuniform in time wISS property with gain $\gamma$ and, thus, the nonuniform in time ISS property, since, according to definition of $\gamma$, for each $t \geq 0$ the function $\gamma(t, \cdot)$ is of class $K_{\infty}$.

ii) $\Rightarrow$ iii) First, we establish that for every $u=u(t)$ of class $C^{0}\left(\left[t_{0},+\infty\right)\right)$ and for every $\left(t_{0}, x_{0}\right) \in \Re^{+} \times \Re^{n}$, the corresponding solution $x(t)$ of (1.1) with $x\left(t_{0}\right)=x_{0}$ exists on $\left[t_{0},+\infty\right)$ and satisfies (3.2) for the same functions $\rho \in K_{\infty}$, $\beta, \phi \in K^{+}$and $\sigma \in K L$ as in statement ii). Notice that for every $u=u(t)$ of class $C^{0}\left(\left[t_{0},+\infty\right)\right)$ there exists a maximal interval, say $\left[t_{0}, T\right)$ for some $T>t_{0}$, of existence of the solution $x(t)$ of (1.1) with $x\left(t_{0}\right)=x_{0}$. By (3.1), it follows, by exploiting continuity of $\phi(t), x(t)$ and $u(t)$, that the solution satisfies the estimate shown in (3.7) at the bottom of the page, where the first, second, and third terms on the right-hand side of (3.7) arise, respectively, for the cases

- $\rho(\phi(\tau)|u(\tau)|) \leq|x(\tau)|$ for all $\tau \in\left[t_{0}, t\right]$;

- $\rho(\phi(t)|u(t)|) \geq|x(t)|$;

- $\rho(\phi(s)|u(s)|) \leq|x(s)|$ for all $s \in[\tau, t]$ for certain $\tau \in\left[t_{0}, t\right]$ which satisfies $\rho(\phi(s)|u(s)|)>|x(s)|$ for $s<\tau$, close to $\tau$ (to be more precise, for the third case, by continuity we obtain $\rho(\phi(\tau)|u(\tau)|)=|x(\tau)|$ and thus by (3.1) $|x(s)| \leq \sigma(\beta(\tau)|x(\tau)|, s-\tau)=$ $\sigma(\beta(\tau) \rho(\phi(\tau)|u(\tau)|), s-\tau)$ for all $s \in[\tau, t]$, hence, $\left.|x(t)| \leq \sup _{t_{0} \leq \tau \leq t} \sigma(\beta(\tau) \rho(\phi(\tau)|u(\tau)|), t-\tau)\right)$.

Notice, by virtue of the right-hand side inequality (3.1), that $s \leq \sigma\left(\beta\left(t_{0}\right) s, 0\right)$, for all $\left(t_{0}, s\right) \in\left(\Re^{+}\right)^{2}$. Consequently, by substituting $s=\rho(\phi(t)|u(t)|)$ and $t_{0}=t$ in the previous inequality, we obtain

$$
\begin{aligned}
\rho(\phi(t)|u(t)|) & \leq \sigma(\beta(t) \rho(\phi(t)|u(t)|), 0) \\
& \leq \sup _{t_{0} \leq \tau \leq t} \sigma(\beta(\tau) \rho(\phi(\tau)|u(\tau)|), t-\tau)
\end{aligned}
$$

hence, by considering the previous three cases, (3.7) is simplified as follows:

$$
\begin{aligned}
& |x(t)| \\
\leq & \max \left\{\sigma\left(\beta\left(t_{0}\right)\left|x_{0}\right|, t-t_{0}\right), \sup _{t_{0} \leq \tau \leq t} \sigma(\beta(\tau) \rho(\phi(\tau)|u(\tau)|), t-\tau)\right\} \\
\forall t \in\left[t_{0}, T\right) . &
\end{aligned}
$$

Estimation (3.8) implies both $T=+\infty$, namely, the solution does not have a finite escape time, and (3.2) is fulfilled for every $u=u(t)$ of class $C^{0}\left(\left[t_{0},+\infty\right)\right)$.

We next show that (3.2) holds for arbitrary $u \in$ $L_{\text {loc }}^{\infty}\left(\left[t_{0},+\infty\right)\right)$. Let $t \in\left[t_{0},+\infty\right), x_{0} \in \Re^{n}$ and $\varepsilon>0$ be arbitrary and define

$$
\begin{aligned}
& r_{1}:=\underset{t_{0} \leq \tau \leq t}{\operatorname{ess} \sup }|u(\tau)| \\
& \tilde{\sigma}(s, \tau):=\sigma(s, \tau)+\frac{s \varepsilon \exp (-\tau)}{2 \max _{t_{0} \leq \xi \leq t} \beta(\xi)\left(1+\rho\left(r_{1} \max _{t_{0} \leq \xi \leq t} \phi(\xi)\right)\right)} \\
& r_{2}:=e s s \sup _{t_{0} \leq \tau \leq t} \tilde{\sigma}(\beta(\tau) \rho(\phi(\tau)|u(\tau)|), t-\tau) \\
& \leq e s s \sup _{t_{0} \leq \tau \leq t} \sigma(\beta(\tau) \rho(\phi(\tau)|u(\tau)|), t-\tau)+\frac{\varepsilon}{2}
\end{aligned}
$$

$$
|x(t)| \leq \max \left\{\sigma\left(\beta\left(t_{0}\right)\left|x_{0}\right|, t-t_{0}\right), \rho(\phi(t)|u(t)|), \sup _{t_{0} \leq \tau \leq t} \sigma(\beta(\tau) \rho(\phi(\tau)|u(\tau)|), t-\tau)\right\} \quad \forall t \in\left[t_{0}, T\right)
$$


Notice that the right-hand side inequality $(3.9 \mathrm{c})$ is an immediate consequence of definitions (3.9a), (3.9b). Moreover, by virtue of definition (3.9b), for every constant $r \geq 0$ there exists a continuous nondecreasing function $\theta_{r}(\cdot)$ such that

$$
\tilde{\sigma}\left(\theta_{r}(t), t\right)=r, \quad \forall t \geq 0 \text {. }
$$

From definitions (3.9a), (3.9c) and property (3.10), it follows that

$$
|u(\tau)| \leq \Theta(\tau):=\min \left\{r_{1}, \frac{1}{\phi(\tau)} \rho^{-1}\left(\frac{\theta_{r_{2}}(t-\tau)}{\beta(\tau)}\right)\right\},
$$$$
\text { for almost all } \tau \in\left[t_{0}, t\right] \text {. }
$$

Since the space $C^{0}\left(\left[t_{0}, t\right]\right)$ is dense in $L^{1}\left(\left[t_{0}, t\right]\right)$, we can find $\tilde{u} \in C^{0}\left(\left[t_{0}, t\right]\right)$ such that

$\|u-\tilde{u}\|_{L^{1}\left(\left[t_{0}, t\right]\right)}=\int_{t_{0}}^{t}|u(\tau)-\tilde{u}(\tau)| d \tau \leq \frac{\varepsilon}{4 L} \exp \left\{-L\left(t-t_{0}\right)\right\}$

where $L$ is any Lipschitz constant for $f(\cdot)$ such that the following hold:

$$
\begin{aligned}
\left|f\left(\tau, y_{1}, v_{1}\right)-f\left(\tau, y_{2}, v_{2}\right)\right| & \leq L\left(\left|y_{1}-y_{2}\right|+\left|v_{1}-v_{2}\right|\right) \\
\forall \tau \in\left[t_{0}, t\right], \quad\left(y_{1}, y_{2}\right) & \in B[0, R] \times B[0, R] \\
\left(v_{1}, v_{2}\right) & \in B\left[0, r_{1}\right] \times B\left[0, r_{1}\right]
\end{aligned}
$$

where $r_{1}$ is defined by (3.9a) and

$$
R:=\sigma\left(\beta\left(t_{0}\right)\left|x_{0}\right|, 0\right)+\sup _{t_{0} \leq \tau \leq t} \sigma\left(\beta(\tau) \rho\left(\phi(\tau) r_{1}\right), 0\right)+2 \varepsilon .
$$

Define

$$
\bar{u}(\tau):=\left\{\begin{array}{ll}
\tilde{u}(\tau), & \text { if }|\tilde{u}(\tau)| \leq \Theta(\tau) \\
\frac{\Theta(\tau)}{|\tilde{u}(\tau)|} \tilde{u}(\tau), & \text { if }|\tilde{u}(\tau)|>\Theta(\tau)
\end{array} .\right.
$$

Notice that definition (3.14) guarantees that $\bar{u} \in C^{0}\left(\left[t_{0}, t\right]\right)$ with $|\bar{u}(\tau)| \leq \Theta(\tau)$ for all $\tau \in\left[t_{0}, t\right]$. In addition, by virtue of definition (3.11), we have $\Theta(\tau) \leq(1 / \phi(\tau)) \rho^{-1}\left(\theta_{r_{2}}(t-\tau) / \beta(\tau)\right)$ for all $\tau \in\left[t_{0}, t\right]$, hence, by virtue of (3.10), it follows that $\tilde{\sigma}(\beta(\tau) \rho(\phi(\tau) \Theta(\tau)), t-\tau) \leq r_{2}$. Consequently, by (3.9)-(3.11) and (3.14), it follows that

$$
\begin{gathered}
|\bar{u}(\tau)| \leq r_{1}=\underset{t_{0} \leq \tau \leq t}{e s s \sup _{1}}|u(\tau)|, \forall \tau \in\left[t_{0}, t\right] \\
\sigma(\beta(\tau) \rho(\phi(\tau)|\bar{u}(\tau)|), t-\tau) \\
\quad \leq \tilde{\sigma}(\beta(\tau) \rho(\phi(\tau) \Theta(\tau)), t-\tau) \\
\leq r_{2} \leq \underset{t_{0} \leq \tau \leq t}{\operatorname{ess} \sup _{0}} \sigma(\beta(\tau) \rho(\phi(\tau)|u(\tau)|), t-\tau)+\frac{\varepsilon}{2} .
\end{gathered}
$$

To be more precise, the first inequality in (3.15b) is a consequence of (3.9b), the second follows from (3.10) and (3.11) and the third one is a consequence of (3.9c). Moreover, definition (3.14), in conjunction with (3.11) guarantees that

$$
\begin{aligned}
|\tilde{u}(\tau)-\bar{u}(\tau)| & =\min \left\{|\tilde{u}(\tau)-v| ;|v| \leq \Theta(\tau), v \in \Re^{m}\right\} \\
& \leq|\tilde{u}(\tau)-u(\tau)|
\end{aligned}
$$

for almost all $\tau \in\left[t_{0}, t\right]$ and therefore $|\tilde{u}-\bar{u}|_{L^{1}\left(\left[t_{0}, t\right]\right)} \leq$ $|u-\tilde{u}|_{L^{1}\left(\left[t_{0}, t\right]\right)}$. This in conjunction with (3.12) gives

$$
\begin{gathered}
\|u-\bar{u}\|_{L^{1}\left(\left[t_{0}, t\right]\right)} \leq\|u-\tilde{u}\|_{L^{1}\left(\left[t_{0}, t\right]\right)}+\|\bar{u}-\tilde{u}\|_{L^{1}\left(\left[t_{0}, t\right]\right)} \\
\leq 2\|u-\tilde{u}\|_{L^{1}\left(\left[t_{0}, t\right]\right)} \leq \frac{\varepsilon}{2 L} \exp \left\{-L\left(t-t_{0}\right)\right\}
\end{gathered}
$$

Let us denote by $x(t)$ and $\bar{x}(t)$ the corresponding solutions of (1.1) with inputs $u$ and $\bar{u}$, respectively, with same initial values $x\left(t_{0}\right)=\bar{x}\left(t_{0}\right)=x_{0}$. By virtue of (3.13b), (3.15a), and the fact that (3.2) holds for every $u=u(t)$ of class $C^{0}\left(\left[t_{0},+\infty\right)\right)$, it follows:

$$
\begin{aligned}
&|\bar{x}(\tau)| \\
& \leq \max \left\{\sigma\left(\beta\left(t_{0}\right)\left|x_{0}\right|, \tau-t_{0}\right), \sup _{t_{0} \leq \xi \leq \tau} \sigma(\beta(\xi) \rho(\phi(\xi)|\bar{u}(\xi)|), \tau-\xi)\right\} \\
&<R \quad \forall \tau \in\left[t_{0}, t\right] .
\end{aligned}
$$

Let $t_{1} \in\left[t_{0}, t\right]$ be the maximal time for which $|x(\tau)| \leq R$ for all $\tau \in\left[t_{0}, t_{1}\right]$, where $R$ is defined by (3.13b). Using Gronwall's inequality, we get by invoking (3.13a), (3.13b), (3.15a), and (3.16): $\sup _{t_{0} \leq \tau \leq t}|x(\tau)-\bar{x}(\tau)| \leq \varepsilon / 2$, for all $\tau \in\left[t_{0}, t_{1}\right]$. This inequality in conjunction with (3.13b), (3.15b), and (3.17) implies

$$
\begin{aligned}
& \quad|x(\tau)| \\
& \leq \max \left\{\sigma\left(\beta\left(t_{0}\right)\left|x_{0}\right|, \tau-t_{0}\right), e s s \sup _{\substack{t_{0} \leq \xi \leq t\\
}} \sigma(\beta(\xi) \rho(\phi(\xi)|u(\xi)|), t-\xi)\right\} \\
& +\varepsilon<R \quad \forall \tau \in\left[t_{0}, t_{1}\right] .
\end{aligned}
$$

Clearly, by virtue of the right-hand side inequality (3.18), it follows that $t_{1}=t$, thus, (3.18) holds for all $\tau \in\left[t_{0}, t\right]$. Consequently, (3.19) holds for $\tau=t$. Obviously, since (3.18) holds for arbitrary $\varepsilon>0$ and $t \in\left[t_{0},+\infty\right)$, we conclude from the first inequality of (3.18) that (3.2) holds for every input $u=u(t)$ of class $u \in L_{\mathrm{loc}}^{\infty}\left(\left[t_{0},+\infty\right)\right)$.

iii) $\Rightarrow$ iv) By invoking Fact $\mathrm{V}$, there exist functions $a \in K_{\infty}$ and $\delta \in K^{+}$such that $\beta(t) \rho(\phi(t)|u|) \leq a(\delta(t)|u|)$ for all $(t, u) \in \Re^{+} \times \Re^{m}$ and if we set $\zeta(s):=\sigma(a(s), 0)+s$ (that obviously is of class $K_{\infty}$ ), the desired (3.3) is a consequence of (3.2) and the previous inequality.

iv) $\Rightarrow$ v) Without loss of generality we may assume that the function $\beta$ involved in (3.3) is nondecreasing. Define $\tilde{\zeta}(s):=$ $\zeta^{-1}((1 / 2) s)$ and let $\theta \in K_{\infty}$ be a locally Lipschitz function that satisfies $\theta(s) \leq \tilde{\zeta}(s)$ for all $s \geq 0$. Then by virtue of (3.3) it follows that for the solution $x(\cdot)$ of $(1.1)$ it holds: $x(\cdot)$

$$
\begin{aligned}
& \delta(\tau)|u(\tau)| \leq \theta(|x(\tau)|) \text { a.e. in }\left[t_{0}, t\right] \\
& \Rightarrow|x(t)| \leq \sigma\left(\beta\left(t_{0}\right)\left|x_{0}\right|, t-t_{0}\right)+\frac{1}{2} \sup _{t_{0} \leq \tau \leq t}|x(\tau)| .
\end{aligned}
$$

Obviously, (3.19) implies

$$
\begin{aligned}
|x(t)| & \leq \sup _{t_{0} \leq \tau \leq t}|x(\tau)| \leq 2 \sigma\left(\beta\left(t_{0}\right)\left|x_{0}\right|, 0\right), \\
\forall t & \geq t_{0}, \text { provided that } \\
\delta(\tau)|u(\tau)| & \leq \theta(|x(\tau)|), \text { a.e. in }\left[t_{0}, t\right] .
\end{aligned}
$$


Notice that every solution $x(\cdot)$ of (3.4) corresponding to some $d \in M_{B[0,1]}$ coincides with the solution of (1.1) corresponding to $u(\cdot)=(\theta(|x(\cdot)|) / \delta(\cdot)) d(\cdot)$ initiated from same initial $x_{0} \in$ $\Re^{n}$ at time $t_{0} \geq 0$. Thus, by taking into account (3.19) and (3.20), it follows that the solution $x(\cdot)$ of (3.4) satisfies:

$$
\begin{aligned}
|x(t)| & \leq \sigma\left(\beta\left(t_{0}\right)\left|x_{0}\right|, t-t_{0}\right)+\frac{1}{2} \sup _{t_{0} \leq \tau \leq t}|x(\tau)|, \\
\forall t & \geq t_{0}, d \in M_{B[0,1]},\left(t_{0}, x_{0}\right) \in \Re^{+} \times \Re^{n} \\
|x(t)| & \leq 2 \sigma\left(\beta\left(t_{0}\right)\left|x_{0}\right|, 0\right), \\
\forall t & \geq t_{0}, d \in M_{B[0,1]},\left(t_{0}, x_{0}\right) \in \Re^{+} \times \Re^{n} .
\end{aligned}
$$

Inequality (3.21b) asserts that $0 \in \Re^{n}$ is robustly stable for (3.4). Next, we establish robust asymptotic stability. Consider the function

$a(t, T, R)$

$$
:=\sup \left\{\left|x\left(t_{0}+t\right)\right| ; d \in M_{B[0,1]},\left|x_{0}\right| \leq R, t_{0} \in[0, T]\right\}
$$

where $x(\cdot)$ denotes the solution of (3.4) corresponding to some $d \in M_{B[0,1]}$. It suffices to show that $\lim _{t \rightarrow+\infty} a(t, T, R)=0$, for all $(T, R) \in\left(\Re^{+}\right)^{2}$. Clearly, by (3.21b) and definition of $a$ we have

$$
a(t, T, R) \leq 2 \sigma(\beta(T) R, 0), \forall t \geq 0 .
$$

Let $\rho:=\varlimsup_{t \rightarrow+\infty} a(t, T, R)$. We show that $\rho=0$. Indeed, for every $\varepsilon>0$ there exists $\tau=\tau(\varepsilon, T, R) \geq 0$ such that

$$
a(t, T, R) \leq \rho+\varepsilon, \quad \forall t \geq \tau .
$$

Again recall definition of $a$ and (3.21a) which imply

$$
\begin{aligned}
a(t, T, R) \leq \sigma(\beta(T+\tau) & a(\tau, T, R), t-\tau) \\
& +\frac{1}{2} \sup _{\tau \leq s \leq t} a(s, T, R), \text { for all } t \geq \tau .
\end{aligned}
$$

This inequality in conjunction with the pair (3.22) and (3.23) gives

$$
\begin{aligned}
\rho & =\varlimsup_{t \rightarrow+\infty} a(t, T, R) \\
& \leq \varlimsup_{t \rightarrow+\infty} \sigma(2 \beta(T+\tau) \sigma(\beta(T) R, 0), t-\tau)+\frac{1}{2} \rho+\frac{1}{2} \varepsilon=\frac{1}{2} \rho+\frac{1}{2} \varepsilon
\end{aligned}
$$

or, equivalently, $\rho \leq \varepsilon$ for all $\varepsilon>0$, which yields $\rho=\lim _{t \rightarrow+\infty} a(t, T, R)=0$. According to definition of the map $a$, this implies attractivity, thus nonuniform in time RGAS of zero with respect to (3.4).

v) $\Rightarrow$ vii) Since $0 \in \Re^{n}$ is (nonuniformly in time) RGAS for (3.4), it follows by the converse Lyapunov theorem in [11], that there exist a $C^{\infty}$ function $V: \Re^{+} \times \Re^{n} \rightarrow \Re^{+}$, functions $a_{1}, a_{2} \in K_{\infty}$ and $\beta \in K^{+}$such that (3.6a) holds and for all $(t, x, d) \in \Re^{+} \times \Re^{n} \times B[0,1]$ we have

$$
\frac{\partial V}{\partial t}(t, x)+\frac{\partial V}{\partial x}(t, x) f\left(t, x, \frac{\theta(|x|)}{\delta(t)} d\right) \leq-V(t, x)
$$

and therefore

$$
\begin{aligned}
& \frac{\partial V}{\partial t}(t, x)+\frac{\partial V}{\partial x}(t, x) f(t, x, u) \leq-V(t, x), \\
& \forall|u| \leq \frac{\theta(|x|)}{\delta(t)},(t, x) \in \Re^{+} \times \Re^{n} .
\end{aligned}
$$

Define (3.25), as shown at the bottom of the page. Clearly, $\psi$ : $\Re^{+} \times \Re^{+} \rightarrow \Re^{+}$is continuous with $\psi(t, 0)=0$ for all $t \geq$ 0 . Consequently, Fact $\mathrm{V}$ guarantees the existence of functions $a_{3} \in K_{\infty}$ and $\phi \in K^{+}$such that $\psi(t, s) \leq a_{3}(\phi(t) s)$.

We next establish inequality (3.6b), with $a_{3}$ as previously, by considering the following two cases.

- $\theta^{-1}(\delta(t)|u|) \leq|x|$. In this case, inequality (3.6b) is a direct consequence of (3.24).

- $\theta^{-1}(\delta(t)|u|) \geq|x|$. In this case, by virtue of definition (3.25) and definition of $a_{3}$, we have

$$
\begin{aligned}
\frac{\partial V}{\partial t}(t, x)+\frac{\partial V}{\partial x}(t, x) f( & t, x, u) \\
& +V(t, x) \leq \psi(t,|u|) \leq a_{3}(\phi(t)|u|)
\end{aligned}
$$

which implies (3.6b).

vii) $\Rightarrow$ ii) Obviously, (3.6a) and (3.6b) imply

$$
\begin{aligned}
\frac{\partial V}{\partial t}(t, x)+ & \frac{\partial V}{\partial x}(t, x) f(t, x, u) \leq-\frac{1}{2} V(t, x) \\
& \forall u \in \Re^{m} \text { with } a_{3}(\phi(t)|u|) \leq \frac{1}{2} a_{1}(|x|) .
\end{aligned}
$$

Define $\rho(s) \quad:=a_{1}^{-1}\left(2 a_{3}(s)\right)$ and $\sigma(s, t) \quad:=$ $a_{1}^{-1}\left(\exp (-t / 2) a_{2}(s)\right)$ and consider the trajectory $x(t)$ of (1.1) that corresponds to some measurable input $u(\cdot)$ for which

$$
\rho(\phi(t)|u(t)|) \leq|x(t)|, \text { a.e. for } t \geq t_{0}
$$

with $\phi$ as defined in (3.6b). Then, (3.26) implies $(d / d t) V(t, x(t)) \leq-(1 / 2) V(t, x(t))$, a.e. for $t \geq t_{0}$, provided that (3.27) holds. The desired (3.1) is a direct consequence of (3.6a), definitions of $\rho$ and $\sigma$ above and previous inequality. Particularly, (3.1) holds with the same $\beta$ and $\phi$ as defined in (3.6a) and (3.6b), respectively.

We finally establish the equivalence between vi) and iv). The implication iv) $\Rightarrow$ vi) is obvious, so we proceed to the establishment of vi) $\Rightarrow$ iv). Suppose that (1.1) satisfies the nonuniform in time 0-GAS property and assume in addition that (3.5) holds. Then, invoking Lemma 2.8, there exist a $C^{\infty}$ map $V: \Re^{+} \times$ $\Re^{n} \rightarrow \Re^{+}$and functions $a_{i} \in K_{\infty}(i=1, \ldots, 4)$ and $p, \kappa \in$ $K^{+}$such that for all $(t, x, u) \in \Re^{+} \times \Re^{n} \times \Re^{m}$ both inequalities

$$
\psi(t, s):=\sup \left\{\frac{\partial V}{\partial t}(t, x)+\frac{\partial V}{\partial x}(t, x) f(t, x, u)+V(t, x) ;|x| \leq \theta^{-1}(\delta(t) s),|u| \leq s\right\} .
$$


(2.5a) and (2.5b) hold. Consider the solution $x(t)$ of (1.1) corresponding to certain $u=u(t)$ of class $L_{\mathrm{loc}}^{\infty}\left(\left[t_{0},+\infty\right)\right)$. Then, by combining (3.5) and (2.5b), it follows that the time derivative of $V$ along the trajectories of (1.1) satisfies

$$
\begin{aligned}
\frac{d}{d t} V(t, x(t)) \leq & -V(t, x(t))+\exp (-2 t) a_{3}\left(2 \sigma\left(\beta\left(t_{0}\right)\left|x_{0}\right|\right)\right) \\
& \cdot \sup _{t_{0} \leq \tau \leq t} a_{4}(\kappa(\tau)|u(\tau)|) \\
& +\exp (-2 t) \sup _{t_{0} \leq \tau \leq t} a_{3}(2 \zeta(\delta(\tau)|u(\tau)|)) \\
& \cdot \sup _{t_{0} \leq \tau \leq t} a_{4}(\kappa(\tau)|u(\tau)|), \text { a.e. for } t \geq t_{0} .
\end{aligned}
$$

We define $\theta_{1}(s):=2\left[a_{3}(2 \zeta(s))+a_{4}(s)\right]^{2}, \bar{\delta}(t):=\kappa(t)+\delta(t)$ and $\theta_{2}(s):=(1 / 2)\left(a_{3}(2 \sigma(s))\right)^{2}$. Clearly, (3.28) in conjunction with previous definitions, implies

$$
\begin{aligned}
& \frac{d}{d t} V(t, x(t)) \leq-V(t, x(t))+\exp (-2 t) \theta_{2}\left(\beta\left(t_{0}\right)\left|x_{0}\right|\right) \\
& +\exp (-2 t) \sup _{t_{0} \leq \tau \leq t} \theta_{1}(\bar{\delta}(\tau)|u(\tau)|), \text { a.e. for } t \geq t_{0}
\end{aligned}
$$

and, therefore, we get

$$
\begin{aligned}
V(t, x(t)) \leq \exp \left(-\left(t-t_{0}\right)\right)\left(V \left(t_{0},\right.\right. & \left.\left.x_{0}\right)+\theta_{2}\left(\beta\left(t_{0}\right)\left|x_{0}\right|\right)\right) \\
& +\sup _{t_{0} \leq \tau \leq t} \theta_{1}(\bar{\delta}(\tau)|u(\tau)|)
\end{aligned}
$$

for every $t \geq t_{0}$. The latter, in conjunction with the left hand-side inequality of (2.5a), implies the desired (3.3) with $\sigma(s, t):=2 a_{1}^{-1}\left(2 \exp (-t)\left(a_{2}(s)+\theta_{2}(s)\right)\right)$, $\beta(t):=p(t)+\beta(t), \delta(t):=\bar{\delta}(t)$ and $\zeta(s):=2 a_{1}^{-1}\left(2 \theta_{1}(s)\right)$. This completes the proof.

Remark 3.3: There are certain links between various gains involved in the equivalent descriptions of nonuniform in time ISS in statements of Proposition 3.1. For instance, if (3.1) holds for certain $\rho \in K_{\infty}, \phi, \beta \in K^{+}$and $\sigma \in K L$, then (3.2) holds with the same $\rho \in K_{\infty}, \phi, \beta \in K^{+}$and $\sigma \in K L$. Moreover, if (3.2) holds for certain $\rho \in K_{\infty}, \phi, \beta \in K^{+}$and $\sigma \in K L$, then (3.3) holds for the same $\beta \in K^{+}$and $\sigma \in K L$. Also note that, if (3.6a),(3.6b) hold for certain pair of functions $\phi, \beta \in$ $K^{+}$, then, according to the analysis made in the establishment of implications vii) $\Rightarrow$ ii) $\Rightarrow$ iii) $\Rightarrow$ iv), it follows that (3.1) and (3.2) hold with same $\phi, \beta \in K^{+}$as above and (3.3) is fulfilled with the same $\beta \in K^{+}$and for certain $\delta \in K^{+}$. To be more precise, the selection of $\delta$ in proof of implication iii) $\Rightarrow$ iv) implies $\delta(t) \geq \phi(t)$ for all $t \geq 0$ away from zero, provided that $\beta \in K_{\infty}$.

Remark 3.4: By exploiting the result of Proposition 3.1 and particularly the equivalence between i) and iii), it can be easily established that the nonuniform in time ISS property remains invariant under the following transformations.

T1) Scaling of time: $\tau \rightarrow t=\int_{0}^{\tau} a(s) d s$ for certain $a \in$ $K^{+}$with $\int_{0}^{+\infty} a(s) d s=+\infty$.

T2) State transformations: $x=\Phi(t, z)$, where $\Phi \in C^{2}\left(\Re^{+} \times \Re^{n} ; \Re^{n}\right)$ satisfying $\Phi(t, 0)=0$, $\operatorname{det}((\partial \Phi / \partial z)(t, z)) \neq 0, \Phi\left(t, \Re^{n}\right)=\Re^{n}$ and $a_{1}(|z|) \leq|\Phi(t, z)| \leq a_{2}(|z|)$, for every $(t, z) \in \Re^{+} \times \Re^{n}$ and for certain $a_{i} \in K_{\infty}(i=1,2)$.
T3) Input transformations: $u=q(t, v)$, where $q \in C^{0}\left(\Re^{+} \times \Re^{m} ; \Re^{m}\right)$ which satisfies $q(t, 0)=0$ for all $t \geq 0$, the map $f(t, x, q(t, v))$ is locally Lipschitz with respect to $v \in \Re^{m}$ and there exists a function $q^{-1} \in C^{0}\left(\Re^{+} \times \Re^{m} ; \Re^{m}\right)$ with $q\left(t, q^{-1}(t, u)\right)=u$ for all $(t, u) \in \Re^{+} \times \Re^{m}$.

The proof of previous statement is immediate and is left to the reader. It should be emphasized here that statement of Remark 3.4 is not in general true for the uniform in time ISS property under T1) and T3).

Finally, an immediate consequence of Proposition 3.1 is the following corollary, which extends the well-known relationship between the notion of uniform in time ISS and the concepts of BIBS and CICS for the autonomous case (see, for instance, [21] and [23]).

Corollary 3.5: Suppose that system (1.1) satisfies the nonuniform in time ISS property and particularly assume that (3.3) holds for certain functions $\zeta \in K_{\infty}, \sigma \in K L, \beta, \delta \in K^{+}$. Let $u \in L_{\text {loc }}^{\infty}\left(\Re^{+}\right)$in such a way that $\delta(t)|u(t)|$ is bounded over $\Re^{+}$. Then for every $\left(t_{0}, x_{0}\right) \in \Re^{+} \times \Re^{n}$, the corresponding solution $x\left(\cdot, t_{0}, x_{0} ; u\right)$ is bounded over $\Re^{+}$and if in addition $\lim _{t \rightarrow+\infty} \delta(t)|u(t)|=0$, then $\lim _{t \rightarrow+\infty} x\left(t, t_{0}, x_{0} ; u\right)=0$.

Proof: Immediate consequence of statement iv) of Proposition 3.1.

Remark 3.6: Corollary 3.5 determines the class of inputs allowed to enter system (1.1) so that its solution remains bounded, or converges to zero, respectively. A better estimation of this class can be made by use of the Lyapunov characterization (3.6) of nonuniform in time ISS. Indeed, (3.6a), (3.6b) imply that for every $\left(t_{0}, x_{0}\right) \in \Re^{+} \times \Re^{n}$ and for every input $u=u(t)$ of class $L_{\mathrm{loc}}^{\infty}\left(\Re^{+}\right)$the corresponding solution $x\left(\cdot, t_{0}, x_{0} ; u\right)$ of (1.1) with initial condition $x\left(t_{0}\right)=x_{0}$, satisfies

$$
\begin{aligned}
a_{1}(|x(t)|) \leq & V(t, x(t)) \leq \exp \left(-\left(t-t_{0}\right)\right) V\left(t_{0}, x_{0}\right) \\
& +\int_{t_{0}}^{t} \exp (-(t-\tau)) a_{3}(\phi(\tau)|u(\tau)|) d \tau
\end{aligned}
$$

from which can be easily deduced that the solution $x\left(\cdot, t_{0}, x_{0} ; u\right)$ is bounded over $\Re^{+}$provided that $\phi(t)|u(t)|$ is bounded over $\Re^{+}$and that $\lim _{t \rightarrow+\infty} x\left(t, t_{0}, x_{0} ; u\right)=0$, if $\lim _{t \rightarrow+\infty} \phi(t)|u(t)|=0$. Notice that according to Remark 3.3, $\delta(t) \geq \phi(t)$ for all $t \geq 0$ away from zero, provided that $\beta \in K_{\infty}$, hence, the Lyapunov characterization (3.6) gives a less conservative estimation of the amplitude of those inputs for which the corresponding solution is bounded, or converges to zero, respectively.

Statement vii) of Proposition 3.1 shows that, under a special type of forward completeness, nonuniform in time 0-GAS Property for (1.1) is equivalent to nonuniform in time ISS for (1.1). For the autonomous case (1.2) we establish below equivalence between nonuniform in time ISS and 0-GAS property, provided that (1.2) is forward complete. It should be pointed out here that, as is shown in [2], the 0-GAS Property plus forward completeness does not in general imply uniform in time ISS. Moreover, since the system is autonomous, the nonuniform in time 0-GAS property is equivalent to the uniform in time 0-GAS property.

Proposition 3.7: Consider the autonomous case (1.2), where $f: \Re^{n} \times \Re^{m} \rightarrow \Re^{n}$ is locally Lipschitz with $f(0,0)=0$. Suppose that i) $0 \in \Re^{n}$ is GAS for the unforced system $\dot{x}=$ 
$f(x, 0)$ (0-GAS Property) and ii) system (1.2) is forward complete. Then, system (1.2) satisfies the nonuniform in time ISS property. Particularly, the solutions of (1.2) satisfy estimates (3.2), (3.3) as in statements iii) and iv), respectively, of Proposition 3.1 with $\beta(t) \equiv 1$.

Proof: Since $0 \in \Re^{n}$ is GAS for system $\dot{x}=f(x, 0)$, then a slight modification of Lemma IV.10 in [2] asserts the existence of a smooth map $V: \Re^{n} \rightarrow \Re^{+}$and functions $a_{1}, a_{2}$, $\lambda, \delta$ of class $K_{\infty}$, such that for all $(x, u) \in \Re^{n} \times \Re^{m}$ we have $a_{1}(|x|) \leq V(x) \leq a_{2}(|x|)$ and $(\partial V / \partial x)(x) f(x, u) \leq$ $-V(x)+\lambda(|x|) \delta(|u|)$. The previous inequalities give the following estimate of the solution $x(\cdot)$ of (1.2) initiated from $x_{0} \in$ $\Re^{n}$ at time $t_{0} \geq 0$ and corresponding to some input $u=u(t)$ of class $L_{\mathrm{loc}}^{\infty}\left(\left[t_{0},+\infty\right)\right)$ :

$$
\begin{aligned}
a_{1}(|x(t)|) \leq & \exp \left(-\left(t-t_{0}\right)\right) a_{2}\left(\left|x_{0}\right|\right) \\
& +\int_{t_{0}}^{t} \exp (-(t-\tau)) \lambda(|x(\tau)|) \delta(|u(\tau)|) d \tau
\end{aligned}
$$$$
\forall t \geq t_{0} \text {. }
$$

Furthermore, since (1.2) is forward complete, [1, Cor. 2.11] guarantees the existence of a smooth and proper map $W: \Re^{n} \rightarrow$ $\Re^{+}$, functions $a_{3}, a_{4}, \sigma$ of class $K_{\infty}$ and a constant $R>0$ such that for all $(x, u) \in \Re^{n} \times \Re^{m}$ it holds $a_{3}(|x|) \leq W(x) \leq$ $a_{4}(|x|)+R$ and $\partial W / \partial x(x) f(x, u) \leq W(x)+\sigma(|u|)$. It then follows that the solution $x(\cdot)$ of (1.2) satisfies

$$
\begin{aligned}
a_{3}(|x(t)|) \leq & \exp \left(t-t_{0}\right)\left(a_{4}\left(\left|x_{0}\right|\right)+R\right) \\
& +\int_{t_{0}}^{t} \exp (t-\tau) \sigma(|u(\tau)|) d \tau, \forall t \geq t_{0} .
\end{aligned}
$$

Define $\tilde{\lambda}(s):=\lambda\left(2 a_{3}^{-1}(2 s)\right)$. It turns out from (3.29) and (3.30) that the following inequality holds for all $t \geq, t_{0}$ :

$$
\begin{aligned}
a_{1}(|x(t)|) \leq & \exp \left(-\left(t-t_{0}\right)\right) a_{2}\left(\left|x_{0}\right|\right) \\
& +\int_{t_{0}}^{t} \tilde{\lambda}\left(\int_{t_{0}}^{\tau} \exp (\tau-s) \sigma(|u(s)|) d s\right) \delta(|u(\tau)|) d \tau \\
& +\int_{t_{0}}^{t} \tilde{\lambda}\left(\exp \left(\tau-t_{0}\right)\left(a_{4}\left(\left|x_{0}\right|\right)+R\right)\right) \delta(|u(\tau)|) d \tau .
\end{aligned}
$$

In order to analyze further estimation (3.31) we first recall Fact II, which guarantees the existence of a function $q \in K_{\infty}$ such that $\sigma(r s)+\delta(r s)+\tilde{\lambda}(r s) \leq q(r) q(s)$, for every $r, s \geq 0$ and let $p$ be a function of class $K^{+}$satisfying $q(1 / p(t)) \leq$ $\exp (-t) / 1+q(\exp (t))$, for all $t \geq 0$. It then follows

$$
\begin{aligned}
& \int_{t_{0}}^{\tau} \exp (\tau-s) \sigma(|u(s)|) d s \\
\leq & \exp (\tau) \sup _{t_{0} \leq s \leq \tau} q(p(s)|u(s)|) \int_{t_{0}}^{\tau} q\left(\frac{1}{p(s)}\right) d s \\
\leq & \exp (\tau) \sup _{t_{0} \leq s \leq \tau} q(p(s)|u(s)|) \quad \forall \tau \geq t_{0}
\end{aligned}
$$

and, thus, by (3.31) and (3.32), (3.33), shown at the bottom of the page, holds. By defining $a(s) \quad:=a_{1}^{-1}\left(2 a_{2}(s)+\left(q\left(2 a_{4}(s)\right)\right)^{2}\right)$ and $\zeta(s) \quad:=$ $a_{1}^{-1}\left(4 q(2 R) q(s)+4(q(s)+q(q(s)))^{2}\right)$, it follows from (3.33) that

$$
|x(t)| \leq a\left(\left|x_{0}\right|\right)+\sup _{t_{0} \leq \tau \leq t} \zeta(p(\tau)|u(\tau)|) \quad \forall t \geq t_{0}
$$

hence, by virture of statement vi) of Proposition 3.1, we conclude that (1.2) satisfies the nonuniform in time ISS property.

Next we prove that (3.2) (statement (iii) of Proposition 3.1) holds with $\beta(t) \equiv 1$. Let $\mu \in K_{\infty}$ and $\gamma \in K^{+}$such that $\exp (2 t) \delta(s) \leq \mu(\gamma(t) s)$, for every $(t, s) \in \Re^{+} \times \Re^{+}$whose existence is guaranteed from Fact V. Exploiting (3.29), (3.34) and previous inequality we obtain (3.35), as shown at the bottom of the page, where $\tilde{a}(s):=s+a_{2}(s)+1 / 2(\lambda(2 a(s)))^{2}$, $\rho(s):=(\mu(s))^{2}+2(\lambda(2 \zeta(s))+\mu(s))^{2}$ and $\phi$ is an appropriate function of class $K^{+} \cap C^{\infty}\left(\Re^{+}\right)$. Obviously, (3.35) implies that the solutions of (1.2) satisfy (3.2) as in statement (iii) of Proposition 3.1 with $\beta(t) \equiv 1$ and $\sigma(s, t):=2 a_{1}^{-1}(2 \exp (-t) \tilde{a}(s))$. It turns out by taking into account Remark 3.3 that statement iv) of Proposition 3.1 as well with $\beta(t) \equiv 1$. This completes the proof.

An immediate consequence of Proposition 3.7 is the following corollary, which provides an equivalent characterization of the 0-GAS property plus forward completeness for autonomous systems (1.2).

$$
\begin{aligned}
a_{1}(|x(t)|) & \leq a_{2}\left(\left|x_{0}\right|\right)+\sup _{t_{0} \leq \tau \leq t} q(p(\tau)|u(\tau)|)\left[\sup _{t_{0} \leq \tau \leq t} q(q(p(\tau)|u(\tau)|))+q\left(a_{4}\left(\left|x_{0}\right|\right)+R\right)\right] \int_{t_{0}}^{t} q(\exp (\tau)) q\left(\frac{1}{p(\tau)}\right) d \tau \\
& \leq a_{2}\left(\left|x_{0}\right|\right)+\sup _{t_{0} \leq \tau \leq t} q(p(\tau)|u(\tau)|)\left[\sup _{t_{0} \leq \tau \leq t} q(q(p(\tau)|u(\tau)|))+q\left(2 a_{4}\left(\left|x_{0}\right|\right)\right)+q(2 R)\right] \quad \forall t \geq t_{0} . \quad(3.33)
\end{aligned}
$$

$$
\begin{aligned}
a_{1}(|x(t)|) \leq & \exp \left(-\left(t-t_{0}\right)\right) a_{2}\left(\left|x_{0}\right|\right)+\exp (-t) \lambda\left(2 a\left(\left|x_{0}\right|\right)\right) \int_{t_{0}}^{t} \exp (-\tau) \mu(\gamma(\tau)|u(\tau)|) d \tau \\
& +\exp (-t) \sup _{t_{0} \leq \tau \leq t} \lambda(2 \zeta(p(\tau)|u(\tau)|)) \int_{t_{0}}^{t} \exp (-\tau) \mu(\gamma(\tau)|u(\tau)|) d \tau \\
\leq & \exp \left(-\left(t-t_{0}\right)\right) \tilde{a}\left(\left|x_{0}\right|\right)+\sup _{t_{0} \leq \tau \leq t} \exp (-(t-\tau)) \tilde{a}(\rho(\phi(\tau)|u(\tau)|)) \quad \forall t \geq t_{0}
\end{aligned}
$$


Corollary 3.8: Consider the autonomous case (1.2), where $f: \Re^{n} \times \Re^{m} \rightarrow \Re^{n}$ is locally Lipschitz with $f(0,0)=0$. Then, (1.2) satisfies the hypotheses i) and ii) of Proposition 3.7, if and only if there exists $\phi \in K^{+} \cap C^{\infty}\left(\Re^{+}\right)$such that (1.3), i.e., system (1.2) with $u=\phi(t) v$, satisfies the uniform in time ISS property from the input $v$.

Proof: If hypotheses i) and ii) of Proposition 3.7 are fulfilled, then statement of the previous proposition asserts that (3.3) holds for certain $\zeta \in K_{\infty}, \delta \in K^{+}, \sigma \in K L$ and $\beta(t) \equiv 1$. Let $\phi \in K^{+} \cap C^{\infty}\left(\Re^{+}\right)$such that $\delta(t) \phi(t) \leq 1$ for all $t \geq 0$. Clearly, system (1.3) satisfies the uniform in time ISS property from the input $v$. The converse statement is obvious. $\triangleleft$

\section{SMALL-GAIN THEOREM}

In this section, we derive sufficient conditions for nonuniform in time ISS for system (1.4), where the mappings $f, g$, are $C^{0}$, locally Lipschitz with respect to $(x, y, u)$ and satisfy $f(t, 0,0,0)=0, g(t, 0,0,0)=0$, for all $t \geq 0$. The next theorem provides a generalization of the small-gain theorem of Jiang-Teel-Praly [7] for the time-varying case (1.4) under the presence of nonuniform in time ISS.

Theorem 4.1: For (1.4), we assume the following.

A1: Subsystem (1.4a) satisfies the nonuniform in time ISS property from the input $(y, u)$. Particularly, assume that there exist $\sigma_{1} \in K L, \rho_{1} \in K_{\infty}, \phi_{1}, \beta_{1} \in K^{+}$such that, for every $\left(t_{0}, x_{0}\right) \in \Re^{+} \times \Re^{n}$ and for every input $(y, u) \in \mathbf{L}_{\mathrm{loc}}^{\infty}\left(\left[t_{0},+\infty\right)\right)$, the solution $x(t)$ of (1.4a) with $x\left(t_{0}\right)=x_{0}$ exists for all $t \geq t_{0}$ and (4.1), as shown at the bottom of the page, holds.

A2: Subsystem (1.4b) satisfies the nonuniform in time ISS property from the input $(x, u)$. Particularly, assume that there exist $\sigma_{2} \in K L, \rho_{2} \in K_{\infty}, \phi_{2}, \beta_{2} \in K^{+}$and a constant $\lambda \geq 0$ such that, for every $\left(t_{0}, y_{0}\right) \in \Re^{+} \times \Re^{k}$ and for every input $(x, u) \in \mathbf{L}_{\text {loc }}^{\infty}\left(\left[t_{0},+\infty\right)\right)$, the solution $y(t)$ of (1.4b) with $y\left(t_{0}\right)=y_{0}$ exists for all $t \geq t_{0}$ and (4.2), as shown at the bottom of the page, holds.

A3: In addition to (4.1) and (4.2) we assume that the following properties hold for all $t_{0}, s \geq 0$ :

$$
\begin{aligned}
\lim _{t \rightarrow+\infty} \beta_{1}(t) \rho_{1}\left(\phi_{1}(t) \sigma_{2}\left(s, t-t_{0}\right)\right) & =0 \\
\lim _{t \rightarrow+\infty} \beta_{2}(t) \rho_{2}\left(\phi_{2}(t) \lambda \sigma_{1}\left(s, t-t_{0}\right)\right) & =0 .
\end{aligned}
$$

A4: Moreover, there exists a function $a$ of class $K_{\infty}$ with

$$
a(s)<s \quad \forall s>0
$$

such that the following inequalities are satisfied for all $t_{0} \geq 0$ :

$$
\begin{aligned}
& \sup _{t \geq t_{0}} \sigma_{1}\left(\beta_{1}(t) \rho_{1}\left(\phi_{1}(t) \sigma_{2}\left(\beta_{2}\left(t_{0}\right) \rho_{2}\left(\phi_{2}\left(t_{0}\right) \lambda s\right), t-t_{0}\right)\right), 0\right) \\
& \quad \leq a(s) \quad \forall s \geq 0 \\
& \sup _{t \geq t_{0}} \sigma_{2}\left(\beta_{2}(t) \rho_{2}\left(\phi_{2}(t) \lambda \sigma_{1}\left(\beta_{1}\left(t_{0}\right) \rho_{1}\left(\phi_{1}\left(t_{0}\right) s\right), t-t_{0}\right)\right), 0\right) \\
& \quad \leq a(s) \quad \forall s \geq 0 .
\end{aligned}
$$

Then, (1.4) satisfies the nonuniform in time ISS property from the input $u$.

Remark 4.2: Obviously, when $\beta_{i}$ and $\phi_{i}(i=1,2)$ are bounded over $\Re^{+}$(case of uniform in time ISS property), then Hypothesis A3 is automatically satisfied. Furthermore, if we define for $i=1,2 r_{i}:=\sup _{t \geq 0} \beta_{i}(t), \mu_{i}:=\sup _{t \geq 0} \phi_{i}(t)$, $\zeta_{i}(s):=\sigma_{i}\left(r_{i} \rho_{i}\left(\mu_{i} s\right), 0\right)$, then it can be easily established that Hypothesis A4 is satisfied as well, provided that

$$
\zeta_{1}\left(\zeta_{2}(\lambda s)\right)<s, \quad \forall s>0
$$

which is exactly the same condition imposed in [7] for the Small-Gain Theorem in the uniform in time case. Indeed, if (4.6) holds, then by virtue of definitions of $r_{i}, \mu_{i}$ and $\zeta_{i}$ above, inequalities (4.5a), (4.5b) are satisfied with $a(s):=\max \left\{\zeta_{1}\left(\zeta_{2}(\lambda s)\right), \zeta_{2}\left(\lambda \zeta_{1}(s)\right),(1 / 2) s\right\}$.

Proof of Theorem 4.1: Let us denote by $\tilde{x}(t):=$ $(x(t), y(t))$ the solution of (1.4) initiated at time $t_{0} \geq 0$ from $\tilde{x}_{0}=\left(x_{0}, y_{0}\right) \in \Re^{n} \times \Re^{k}$ and corresponding to some input $u \in \mathbf{L}_{\mathrm{loc}}^{\infty}\left(\left[t_{0},+\infty\right)\right)$. The following claim is proved in the Appendix and provides essential estimates for the solution of (1.4).

Claim: Under hypotheses A1-A4, there exist functions $B_{i} \in$ $K L, \tilde{\beta}_{i} \in K^{+}(i=1,2), \zeta_{i} \in K_{\infty}$ and $\tilde{\delta}_{i} \in K^{+}(i=1,2)$ such that the following estimates hold for the solution $\tilde{x}(t)$ of (1.4) for all $t \geq t_{0}$; see (4.7a)-(4.8b), as shown at the bottom of the next page, where $a$ is defined in (4.4).

Next, we prove that under hypotheses A1-A4, system (1.4) satisfies the nonuniform in time 0-GAS property. Without loss of generality we may assume that the functions $\tilde{\beta}_{i} i=1,2$

$$
|x(t)| \leq \max \left\{\sigma_{1}\left(\beta_{1}\left(t_{0}\right)\left|x_{0}\right|, t-t_{0}\right), \sup _{t_{0} \leq \tau \leq t} \sigma_{1}\left(\beta_{1}(\tau) \rho_{1}\left(\phi_{1}(\tau) \max \{|y(\tau)|,|u(\tau)|\}\right), t-\tau\right)\right\} .
$$

$$
|y(t)| \leq \max \left\{\sigma_{2}\left(\beta_{2}\left(t_{0}\right)\left|y_{0}\right|, t-t_{0}\right), \sup _{t_{0} \leq \tau \leq t} \sigma_{2}\left(\beta_{2}(\tau) \rho_{2}\left(\phi_{2}(\tau) \max \{\lambda|x(\tau)|,|u(\tau)|\}\right), t-\tau\right)\right\} .
$$


[involved in (4.7a), (4.7b) and (4.8a), (4.8b)] are both nondecreasing. Consider the solution $\tilde{x}(t)=(x(t), y(t))$ of (1.4) initiated at time $t_{0} \geq 0$ from $\tilde{x}_{0}=\left(x_{0}, y_{0}\right) \in \Re^{n} \times \Re^{k}$ and corresponding to zero input $u(\cdot) \equiv 0$. Using the estimates (4.8a), (4.8b) we get

$$
|\tilde{x}(t)| \leq B_{1}\left(\tilde{\beta}_{1}\left(t_{0}\right)\left|\tilde{x}_{0}\right|, 0\right)+B_{2}\left(\tilde{\beta}_{2}\left(t_{0}\right)\left|\tilde{x}_{0}\right|, 0\right) \quad \forall t \geq t_{0} .
$$

By (4.9) it follows that the origin for system (1.4) with $u \equiv 0$ is (nonuniformly in time) stable. Next, we establish asymptotic stability. Consider the following functions defined for $\tau \geq 0$, $T \geq 0$, and $s \geq 0$ :

$$
\begin{aligned}
& \chi_{1}(\tau, T, s):=\sup \left\{\left|x\left(t_{0}+\tau\right)\right| ;\left|\tilde{x}_{0}\right| \leq s, t_{0} \in[0, T]\right\} \\
& \chi_{2}(\tau, T, s):=\sup \left\{\left|y\left(t_{0}+\tau\right)\right| ;\left|\tilde{x}_{0}\right| \leq s, t_{0} \in[0, T]\right\} .
\end{aligned}
$$

In order to establish asymptotic stability, it suffices to show that $\lim _{\tau \rightarrow+\infty} \chi_{i}(\tau, T, s)=0$ for $i=1$, 2. Clearly, by virtue of (4.9), both $\chi_{i}(\tau, T, s)$ are bounded, thus $\varlimsup_{\tau \rightarrow+\infty} \chi_{i}(\tau, T, s)=l_{i}<$ $+\infty$ for $i=1,2$. It turns out that for every $\varepsilon>0$ there exists $\xi:=\xi(\varepsilon, T, s)>0$ such that for $i=1,2$ it holds

$$
l_{i} \leq \sup _{\tau \geq \xi} \chi_{i}(\tau, T, s) \leq l_{i}+\varepsilon
$$

Exploiting (4.7a), (4.7b) with $u \equiv 0$ we get (4.12a)-(4.12b), as shown at the bottom of the page. By (4.10), (4.11), and (4.12a), (4.12b), it then follows that

$$
l_{i} \leq a\left(l_{i}+\varepsilon\right), \quad i=1,2 .
$$

However, we have assumed in (4.4) that $a(s)<s$ for all $s>0$ and since $\varepsilon>0$ is arbitrary, we conclude from (4.13) that $l_{i}={\overline{\lim _{t \rightarrow+\infty}}} \chi_{i}(t, T, s)=0$ for $i=1,2$, thus system (1.4) satisfies the nonuniform in time 0 -GAS property. This fact in conjunction with estimates (4.8a), (4.8b) asserts that statement vi) of Proposition 3.1 is fulfilled and thus (1.4) satisfies the nonuniform in time ISS property. The proof is complete.

For the case of cascade systems (1.5) we obtain the following result, which constitutes a generalization of recent results obtained in [18] and [32] concerning time-varying systems.

Corollary 4.3: For the system (1.5) we assume that Hypothesis A1 of Theorem 4.1 holds and subsystem (1.5b) satisfies the nonuniform in time ISS property from the input $u$; particularly, there exist $\sigma_{2} \in K L, \rho_{2} \in K_{\infty}, \phi_{2}, \beta_{2} \in K^{+}$, such that, for every $\left(t_{0}, y_{0}\right) \in \Re^{+} \times \Re^{k}$ and for every input $u \in$ $\mathbf{L}_{\text {loc }}^{\infty}\left(\left[t_{0},+\infty\right)\right)$, the solution $y(t)$ of $(1.5 \mathrm{~b})$ with $y\left(t_{0}\right)=y_{0}$ exists for all $t \geq t_{0}$ and satisfies the equation shown bottom of the page. Moreover, it holds $\lim _{t \rightarrow+\infty} \beta_{1}(t) \rho_{1}\left(\phi_{1}(t) \sigma_{2}\left(s, t-t_{0}\right)\right)=$ 0 for all $t_{0}, s \geq 0$. Then, $(1.5)$ satisfies the nonuniform in time ISS property.

Proof: It is an immediate application of Theorem 4.1 with $\lambda=0$.

$$
\begin{aligned}
& |x(t)| \leq \max \left\{B_{1}\left(\tilde{\beta}_{1}\left(t_{0}\right)\left|\tilde{x}_{0}\right|, t-t_{0}\right), a\left(\sup _{t_{0} \leq \tau \leq t}|x(\tau)|\right), \sup _{t_{0} \leq \tau \leq t} \zeta_{1}\left(\tilde{\delta}_{1}(\tau)|u(\tau)|\right)\right\} \\
& |y(t)| \leq \max \left\{B_{2}\left(\tilde{\beta}_{2}\left(t_{0}\right)\left|\tilde{x}_{0}\right|, t-t_{0}\right), a\left(\sup _{t_{0} \leq \tau \leq t}|y(\tau)|\right), \sup _{t_{0} \leq \tau \leq t} \zeta_{2}\left(\tilde{\delta}_{2}(\tau)|u(\tau)|\right)\right\} \\
& |x(t)| \leq \max \left\{B_{1}\left(\tilde{\beta}_{1}\left(t_{0}\right)\left|\tilde{x}_{0}\right|, 0\right), \sup _{t_{0} \leq \tau \leq t} \zeta_{1}\left(\tilde{\delta}_{1}(\tau)|u(\tau)|\right)\right\} \\
& |y(t)| \leq \max \left\{B_{2}\left(\tilde{\beta}_{2}\left(t_{0}\right)\left|\tilde{x}_{0}\right|, 0\right), \sup _{t_{0} \leq \tau \leq t} \zeta_{2}\left(\tilde{\delta}_{2}(\tau)|u(\tau)|\right)\right\}
\end{aligned}
$$

$$
\begin{array}{ll}
|x(t)| \leq \max \left\{B_{1}\left(\tilde{\beta}_{1}\left(t_{0}+\xi\right)\left|\tilde{x}\left(t_{0}+\xi\right)\right|, t-\xi-t_{0}\right), a\left(\sup _{\xi \leq \tau \leq t-t_{0}}\left|x\left(t_{0}+\tau\right)\right|\right)\right\} & \\
|y(t)| \leq \max \left\{B_{2}\left(\tilde{\beta}_{2}\left(t_{0}+\xi\right)\left|\tilde{x}\left(t_{0}+\xi\right)\right|, t-\xi-t_{0}\right), a\left(\sup _{\xi \leq \tau \leq t-t_{0}}\left|y\left(t_{0}+\tau\right)\right|\right)\right\} & \forall t \geq t_{0}+\xi .
\end{array}
$$

$$
|y(t)| \leq \max \left\{\sigma_{2}\left(\beta_{2}\left(t_{0}\right)\left|y_{0}\right|, t-t_{0}\right), \sup _{t_{0} \leq \tau \leq t} \sigma_{2}\left(\beta_{2}(\tau) \rho_{2}\left(\phi_{2}(\tau)|u(\tau)|\right), t-\tau\right)\right\} .
$$




\section{APPLICATIONS AND NUMERICAL EXAMPLES}

In this section, we provide some applications and numerical examples by exploiting the result of the Small-Gain Theorem 4.1. The first Example 5.1 deals with the problem of output feedback stabilization of (1.6) and constitutes a generalization of the corresponding result in [7].

Example 5.1: We first apply the result of Corollary 3.8 and Theorem 4.1 for the output feedback stabilization for the autonomous case (1.6), where $y$ is the output of the system. We establish that, under hypotheses (1.7a)-(1.7c), there exists a smooth time-varying output feedback $u=k(t, y)$ with $k(\cdot, 0)=0$ that guarantees nonuniform in time ISS for the resulting system (1.6) with $u=k(t, y)+v$ from the input $v \in \Re$. The procedure is similar to that employed in [7], however a more careful analysis is needed here. First, by taking into account the result of Proposition 3.7 it follows that there exist functions $\phi \in K^{+} \cap C^{\infty}\left(\Re^{+}\right), \rho_{1} \in K_{\infty}$ and $\sigma_{1} \in K L$ such that, for every $\left(t_{0}, x_{0}\right) \in \Re^{+} \times \Re^{n}$ and for every input $y \in L_{\mathrm{loc}}^{\infty}\left(\left[t_{0},+\infty\right)\right)$, the corresponding solution $x(t)$ of system (1.6a) with $x\left(t_{0}\right)=x_{0}$, exists for all $t \geq t_{0}$ and satisfies

$$
|x(t)| \leq \max \left\{\sigma_{1}\left(\left|x_{0}\right|, t-t_{0}\right), \sup _{t_{0} \leq \tau \leq t} \sigma_{1}\left(\rho_{1}(|\phi(\tau) y(\tau)|), t-\tau\right)\right\} .
$$

Without loss of generality, we may assume that $\phi(t) \geq 1$, for all $t \geq 0$.

Claim 1: The functions $\sigma_{1} \in K L$ and $\rho_{1} \in K_{\infty}$ involved in (5.1) can be selected in such a way that $\rho_{1}(s):=s$ for all $s \geq 0$ and $\sigma_{1}(s, t)=K s \exp (-l t)$ for all $(t, s) \in \Re^{+} \times[0, r]$ for certain constants $K, l, r>0$.

Proof of Claim 1: The proof is based on our hypothesis (1.7c), namely, that the matrix $A:=(\partial f / \partial x)(0,0)$ is Hurwitz and can be made by standard arguments like those for the time-invariant case (see for instance [7], [20]). Assumption $(1.7 \mathrm{c})$, guarantees that there exist constants $K, l, r>0$ such that, for every $\left(t_{0}, x_{0}\right) \in \Re^{+} \times B[0,2 r], t \geq t_{0}$ and input $y \in L_{\mathrm{loc}}^{\infty}\left(\left[t_{0},+\infty\right)\right)$ with $\sup _{t_{0} \leq \tau \leq t}|y(\tau)| \leq 2 r$, the corresponding solution $x(t)$ of system (1.6a) with $x\left(t_{0}\right)=x_{0}$ satisfies

$$
|x(t)| \leq K \exp \left(-l\left(t-t_{0}\right)\right)\left[\left|x_{0}\right|+\sup _{t_{0} \leq \tau \leq t}(\exp (l \tau)|y(\tau)|)\right]
$$

We next combine (5.1) and (5.2) to reconstruct the functions $\rho_{1} \in K_{\infty}$ and $\sigma_{1} \in K L$ in such a way that (5.1) holds and simultaneously the rest desired properties of our claim are fulfilled. Notice first, by recalling Facts III and V, that there exist functions $a_{1}, a_{2} \in K_{\infty}, \tilde{a}_{2} \in K_{\infty}$ and $\tilde{\phi} \in K^{+}$so that $\sigma_{1}(s, t) \leq a_{1}^{-1}\left(\exp (-t) a_{2}(s)\right)$ and $\exp (t) a_{2}\left(\rho_{1}(\phi(t) s)\right) \leq$ $\tilde{a}_{2}(\tilde{\phi}(t) s)$ for all $s, t \geq 0$, hence, by defining $\tilde{\sigma}_{1}(s, t):=$ $a_{1}^{-1}\left(\exp (-t) \max \left\{a_{2}(s), \tilde{a}_{2}(s)\right\}\right)$, inequality (5.1) implies

$$
|x(t)| \leq \tilde{\sigma}_{1}\left(\left|x_{0}\right|+\sup _{t_{0} \leq \tau \leq t}(\tilde{\phi}(\tau)|y(\tau)|), t-t_{0}\right) .
$$

By combining (5.2) and (5.3) we can construct mappings $\phi \in$ $K^{+} \cap C^{\infty}\left(\Re^{+}\right)$and $\sigma \in K L$ with $\phi(t) \geq \max \{\tilde{\phi}(t), \exp (l t)\}$ for all $t \geq 0$ and $\sigma(s, t) \geq \tilde{\sigma}_{1}(s, t)$ for all $(t, s) \in \Re^{+} \times$ $[2 r,+\infty)$ and in such a way that $\sigma(s, t)=K s \exp (-l t)$ for all $(t, s) \in \Re^{+} \times[0, r]$ and furthermore, for every $\left(t_{0}, x_{0}\right) \in \Re^{+} \times$ $\Re^{n}$ and for every input $y \in L_{\mathrm{loc}}^{\infty}\left(\left[t_{0},+\infty\right)\right)$, the corresponding solution $x(t)$ of (1.6a) with $x\left(t_{0}\right)=x_{0}$ satisfies

$$
\begin{aligned}
& |x(t)| \\
\leq & \sigma\left(\left|x_{0}\right|+\sup _{t_{0} \leq \tau \leq t}(\phi(\tau)|y(\tau)|), t-t_{0}\right) \\
\leq & \sigma\left(2\left|x_{0}\right|, t-t_{0}\right)+\sigma\left(\sup _{t_{0} \leq \tau \leq t}(2 \phi(\tau)|y(\tau)|), t-t_{0}\right) \\
\leq & \max \left\{2 \sigma\left(2\left|x_{0}\right|, t-t_{0}\right), \sup _{t_{0} \leq \tau \leq t} 2 \sigma\left((2 \phi(\tau)|y(\tau)|), t-t_{0}\right)\right\} \\
\leq & \max \left\{2 \sigma\left(2\left|x_{0}\right|, t-t_{0}\right), \sup _{t_{0} \leq \tau \leq t} 2 \sigma((2 \phi(\tau)|y(\tau)|), t-\tau)\right\}
\end{aligned}
$$

The last inequality shown previously proves Claim 1 . Particularly, (5.1) holds with $\sigma_{1}(s, t):=2 \sigma(2 s, t), \rho_{1}(s) \equiv s$ and further the rest requirement for $\sigma_{1}$ is fulfilled.

By taking into account properties of $\sigma_{1}$ and $\rho_{1}$ in Claim 1 we can determine a pair of locally Lipschitz functions $\zeta, \rho_{2} \in K_{\infty}$ being linear near zero such that

$$
\zeta(s) \geq \sigma_{1}\left(\rho_{1}(s), 0\right) \quad \text { and } \quad \rho_{2}(s):=\frac{1}{2} \zeta^{-1}\left(\frac{s}{2}\right) .
$$

In order to simplify the rest of the analysis, we first consider the auxiliary one-dimensional system

$$
\begin{aligned}
& \dot{z}=\phi(t) g\left(x, \frac{z}{\phi(t)}\right)+\frac{\dot{\phi}(t)}{\phi(t)} z+\phi(t) h(t, z)+\phi(t) v \\
& z \in \Re, t \in \Re^{+} \text {with }(x, v) \in \Re^{n} \times \Re \text { as input }
\end{aligned}
$$

and prove the following claim.

Claim 2: There exists a $C^{\infty}$ function $h: \Re^{+} \times \Re \rightarrow \Re$ with $h(\cdot, 0)=0$, such that for every $\left(t_{0}, z_{0}\right) \in \Re^{+} \times \Re$ and for every input $(x, v) \in L_{\mathrm{loc}}^{\infty}\left(\left[t_{0},+\infty\right)\right)$, the solution $z(t)$ of (5.5) with $z\left(t_{0}\right)=z_{0}$ exists for all $t \geq t_{0}$ and satisfies (5.6), as shown at the bottom of the page, where $\rho_{2}$ is defined by (5.4).

Proof of Claim 2: We prove that there exists a $C^{\infty}$ function $h: \Re^{+} \times \Re \rightarrow \Re$ with $h(\cdot, 0)=0$ such that for every

$$
|z(t)| \leq \max \left\{\exp \left(-\left(t-t_{0}\right)\right)\left|z_{0}\right|, \sup _{t_{0} \leq \tau \leq t} \exp (-(t-\tau)) \rho_{2}(\exp (-\tau) \max \{|x(\tau)|,|v(\tau)|\})\right\}, \quad \forall t \geq t_{0}
$$


$\left(t_{0}, z_{0}\right) \in \Re^{+} \times \Re$ and for every input $(x, v) \in L_{\mathrm{loc}}^{\infty}\left(\left[t_{0},+\infty\right)\right)$ the solution $z(t)$ of (5.5) with $z\left(t_{0}\right)=z_{0}$ exists for all $t \geq t_{0}$ and satisfies

$$
\begin{aligned}
& \rho_{2}\left(\frac{\exp (-t)}{\sqrt{2}}|(x, v)|\right) \leq|z(t)| \\
& \quad \text { a.e for } t \geq t_{0} \Rightarrow|z(t)| \leq \exp \left(-\left(t-t_{0}\right)\right)\left|z_{0}\right|
\end{aligned}
$$

Define $\tilde{g}(t, x, z, v):=\phi(t) g(x, z / \phi(t))+\dot{\phi}(t) / \phi(t) z+\phi(t) v$ and notice, by using Fact $\mathrm{V}$, that there exist functions $a \in K_{\infty}$, $q \in K^{+}$, such that for every $(t, z) \in \Re^{+} \times \Re$, it holds that $\sup \left\{|\tilde{g}(t, x, z, v)| ;|(x, v)| \leq \sqrt{2} \exp (t) \rho_{2}^{-1}(|z|)\right\} \leq$ $a(q(t)|z|)$. Since $\tilde{g}(t, x, z, v)$ is locally Lipschitz with respect to $(x, z, v) \in \Re^{n} \times \Re \times \Re$ and $\rho_{2}^{-1}(s)$ is linear near zero, we may assume that $a \in K_{\infty}$ and $q \in K^{+}$are both of class $C^{\infty}\left(\Re^{+}\right)$and that the function $a \in K_{\infty}$ can be selected in such a way that is linear near zero. Let $\tilde{a}: \Re \rightarrow \Re$ be the odd extension of $a$. Then, $\tilde{a}: \Re \rightarrow \Re$ is of class $C^{\infty}(\Re)$ and satisfies $|z||\tilde{g}(t, x, z, v)| \leq z \tilde{a}(q(t) z)$ for every $|(x, v)| \leq \sqrt{2} \exp (t) \rho_{2}^{-1}(|z|), t \in \Re^{+}$and $z \in \Re$. By defining $h(t, z):=-\tilde{a}(q(t) z)-z$ and $V(t, z):=z^{2} / 2$ and taking into account that $\phi(t) \geq 1$ for all $t \geq 0$, it follows that the derivative $\dot{V}$ of the function $V$ along the trajectories of (5.5) satisfies: $\dot{V} \leq-2 V$ for $|(x, v)| \leq \sqrt{2} \exp (t) \rho_{2}^{-1}(|z|)$, $t \in \Re^{+}$and $z \in \Re$. The previous property implies, according to equivalence of statements iii) and iv) of Proposition 2.5, the desired (5.7). Property (5.7), according to the equivalence between statements ii) and iii) of Proposition 3.1, implies (5.6). Precisely, in order to derive (5.6), we invoke implication ii) $\Rightarrow$ iii) of Proposition 3.1 together with Remark 3.3 and use the elementary inequality $|(x, v)| \leq \sqrt{2} \max \{|x|,|v|\}$.

We next apply the nonuniform Small-Gain Theorem 4.1 for the following composite system:

$$
\begin{aligned}
& \dot{x}=f\left(x, \frac{z}{\phi(t)}\right) \\
& \dot{z}=\phi(t) g\left(x, \frac{z}{\phi(t)}\right)+\frac{\dot{\phi}(t)}{\phi(t)} z+\phi(t) h(t, z)+\phi(t) v \\
& z \in \Re, v \in \Re, x \in \Re^{n}, t \in \Re^{+} .
\end{aligned}
$$

Inequalities (5.1) and (5.6) assert that hypotheses A1 and A2 of Theorem 4.1 are fulfilled with $\beta_{1}(t) \equiv 1, \phi_{1}(t) \equiv 1, \beta_{2}(t) \equiv 1$, $\phi_{2}(t):=\exp (-t), \lambda=1$ and $\sigma_{2}(s, t):=s \exp (-t)$. Moreover, definition (5.4) of $\rho_{2}$ guarantee

$$
\begin{aligned}
\lim _{t \rightarrow+\infty} \rho_{1}\left(s \exp \left(-\left(t-t_{0}\right)\right)\right) & =0 \\
\lim _{t \rightarrow+\infty} \rho_{2}\left(\exp (-t) \sigma_{1}\left(s, t-t_{0}\right)\right) & =0 \\
\sup _{t \geq t_{0}} \zeta\left(\rho_{2}\left(\exp \left(-t_{0}\right) s\right) \exp \left(-\left(t-t_{0}\right)\right)\right) & \leq \frac{1}{2} s \\
\sup _{t \geq t_{0}} \rho_{2}(\exp (-t) \zeta(s)) \leq \frac{1}{2} s \quad \forall s, t_{0} & \geq 0 .
\end{aligned}
$$

Inequalities (5.10) show that Hypothesis A4 of Theorem 4.1 holds with $a(s):=s / 2<s$ and properties (5.9) show that Hypothesis A3 of same theorem is fulfilled as well, thus, Theorem 4.1 asserts that system (5.8) satisfies the nonuniform in time ISS property from the input $v$. Therefore, according to Proposition 3.1, there exist functions $\bar{\beta}, \bar{\phi} \in K^{+}, \bar{\rho} \in K_{\infty}$ and $\bar{\rho} \in K L$ such that the solution of (5.8) satisfies the estimate shown in (5.11) at the bottom of the page. We finally apply the reverse transformation $y=z / \phi(t)$ and system (5.8) takes the original form: $\dot{x}=f(x, y), \dot{y}=g(x, y)+h(t, \phi(t) y)+v$. It follows from (5.11) and by taking into account that $\phi(t) \geq 1$ for all $t \geq 0$ that (5.12), as shown at the bottom of the page, holds. Hence, we conclude that (1.6) with $u=k(t, y)+v$, $k(t, y):=h(t, \phi(t) y)$ satisfies the nonuniform in time ISS property from the input $v$.

We next derive sufficient conditions for nonuniform in time ISS for composite linear systems (1.8) and provide a numerical example.

Example 5.2: Consider the linear time-varying system (1.8). According to the analysis made in Example 2.9, the following statements are equivalent.

- System (1.8) satisfies the nonuniform in time ISS property.

- The origin $0 \in \Re^{n+k}$ is nonuniformly in time GAS for (1.8) with $u \equiv 0$, namely, with respect to

$$
\begin{aligned}
& \dot{x}=A_{11}(t) x+A_{12}(t) y \\
& \dot{y}=A_{21}(t) x+A_{22}(t) y \\
& x \in \Re^{n}, y \in \Re^{k}, t \in \Re^{+} .
\end{aligned}
$$

We next establish that the origin is nonuniformly in time GAS for system (5.13) under the following assumptions:

$$
|(x(t), z(t))| \leq \max \left\{\bar{\sigma}\left(\bar{\beta}\left(t_{0}\right)\left|\left(x_{0}, z_{0}\right)\right|, t-t_{0}\right), \sup _{t_{0} \leq \tau \leq t} \bar{\sigma}(\bar{\beta}(\tau) \bar{\rho}(\bar{\phi}(\tau)|v(\tau)|), t-\tau)\right\}, \quad \forall t \geq t_{0}
$$

$$
|(x(t), y(t))| \leq \max \left\{\bar{\sigma}\left(\bar{\beta}\left(t_{0}\right) \phi\left(t_{0}\right)\left|\left(x_{0}, y_{0}\right)\right|, t-t_{0}\right), \sup _{t_{0} \leq \tau \leq t} \bar{\sigma}(\bar{\beta}(\tau) \phi(\tau) \bar{\rho}(\bar{\phi}(\tau)|v(\tau)|), t-\tau)\right\}, \quad \forall t \geq t_{0}
$$


H1: There exist functions $\phi_{i}, \mu_{i}$ and $\beta_{i} \in K^{+}(i=1,2)$ with $\int_{0}^{+\infty} \mu_{1}(t) d t=\int_{0}^{+\infty} \mu_{2}(t) d t=+\infty$ such that the solution $(x(t), y(t))$ of (5.13) satisfies

$$
\begin{aligned}
\phi_{1}(\tau)|y(\tau)| \leq & |x(\tau)| \text { a.e. for } \tau \in\left[t_{0}, t\right] \\
\Rightarrow & |x(t)| \leq \exp \left(-\int_{0}^{t-t_{0}} \mu_{1}(s) d s\right) \\
& \cdot \beta_{1}\left(t_{0}\right)\left|x_{0}\right| \\
\phi_{2}(\tau)|x(\tau)| \leq & |y(\tau)| \text { a.e. for } \tau \in\left[t_{0}, t\right] \\
\Rightarrow & |y(t)| \leq \exp \left(-\int_{0}^{t-t_{0}} \mu_{2}(s) d s\right) \\
& \cdot \beta_{2}\left(t_{0}\right)\left|y_{0}\right| .
\end{aligned}
$$

H2: The functions $\phi_{i}, \beta_{i}$ and $\mu_{i}$ satisfy

$\lim _{t \rightarrow+\infty} \beta_{1}(t) \phi_{1}(t) \exp \left(-\int_{0}^{t-t_{0}} \mu_{2}(s) d s\right)=0$

$\lim _{t \rightarrow+\infty} \beta_{2}(t) \phi_{2}(t) \exp \left(-\int_{0}^{t-t_{0}} \mu_{1}(s) d s\right)=0, \quad \forall t_{0} \geq 0$.

H3: In addition to (5.15), it holds that

$$
\begin{aligned}
\sup _{t_{0} \geq 0} \gamma_{1}\left(t_{0}\right) \gamma_{2}\left(t_{0}\right) & <1 \\
\gamma_{1}\left(t_{0}\right) & :=\sup _{t \geq t_{0}} \beta_{1}(t) \phi_{1}(t) \exp \left(-\int_{0}^{t-t_{0}} \mu_{2}(s) d s\right) \\
\gamma_{2}\left(t_{0}\right) & :=\sup _{t \geq t_{0}} \beta_{2}(t) \phi_{2}(t) \exp \left(-\int_{0}^{t-t_{0}} \mu_{1}(s) d s\right) .
\end{aligned}
$$

Inequality (5.16a) is a generalization of the familiar smallgain condition for autonomous linear systems (see, for instance, [14]). Notice also that, by virtue of Example 2.8, Hypothesis H1 is equivalent to the assumption that zero is nonuniformly in time GAS for the systems $\dot{x}=A_{11}(t) x$ and $\dot{y}=A_{22}(t) y$. Moreover, by invoking Remark 3.3, it follows that Assumption $\mathrm{H} 1$ implies both Hypotheses A1 and A2 of Small-Gain Theorem 4.1. Assumption $\mathrm{H} 2$ also guarantees that Hypothesis $\mathrm{A} 3$ of Theorem 4.1 holds and further, by virtue of Assumption $\mathrm{H} 3$, there exists a constant $L \in(0,1)$ such that

$$
\begin{array}{r}
\beta_{2}\left(t_{0}\right) \phi_{2}\left(t_{0}\right) \sup _{t \geq t_{0}} \beta_{1}(t) \phi_{1}(t) \\
\exp \left(-\int_{0}^{t-t_{0}} \mu_{2}(s) d s\right) \leq L \quad \text { (5.17a) } \\
\beta_{1}\left(t_{0}\right) \phi_{1}\left(t_{0}\right) \sup _{t \geq t_{0}} \beta_{2}(t) \phi_{2}(t) \exp \left(-\int_{0}^{t-t_{0}} \mu_{1}(s) d s\right) \leq L, \\
\forall t_{0} \geq 0 . \quad(5.17 \mathrm{~b})
\end{array}
$$

Inequalities (5.17a), (5.17b) guarantee that Hypothesis A4 of Theorem 4.1 holds as well with $a(s):=L s$. We conclude, according to the statement of Theorem 4.1, that zero is nonuniformly in time GAS for (5.13); equivalently, (1.8) satisfies the nonuniform in time ISS property.
We illustrate the nature of the previous hypotheses (5.14a), (5.14b), (5.15) and (5.16a), (5.16b) by considering the following numerical example. Consider the planar system

$$
\begin{aligned}
& \dot{x}=-x+a_{1} \exp (-t) y \\
& \dot{y}=a_{2}(1+t) x-K y
\end{aligned}
$$

where $a_{i}(i=1,2)$ and $K>0$ are constants satisfying

$$
\left|a_{1} a_{2}\right|<\frac{K}{8} \exp \left(\frac{3}{2}\right) .
$$

We show that hypotheses (5.14a), (5.14b), (5.15), and (5.16a), (5.16b) hold, thus, zero is nonuniformly in time GAS with respect to (5.19). Let $\varepsilon_{1} \in(0,1)$ and $\delta>0$ yet to be specified and let $V(x):=(1 / 2) x^{2}$ and $\phi_{1}(t):=\left(\left(\delta+\left|a_{1}\right|\right) / \varepsilon_{1}\right) \exp (-t)$. We evaluate the derivative $\dot{V}$ of $V$ along the trajectories of the subsystem (5.18a). We find $\dot{V}=-x^{2}+a_{1} \exp (-t) x y \leq$ $-x^{2}+\left(\mid a_{1}+\delta\right) \exp (-t)|x \| y|$ and this (by virtue of Proposition 2.5) implies

$$
\begin{aligned}
\phi_{1}(s)|y(s)| & \leq|x(s)| \text { a.e for } s \in\left[t_{0}, t\right] \\
& \Rightarrow|x(t)| \leq \exp \left(-\left(1-\varepsilon_{1}\right)\left(t-t_{0}\right)\right)\left|x_{0}\right| .
\end{aligned}
$$

Likewise, by setting $W(y):=(1 / 2) y^{2}$ and $\phi_{2}(t):=((\delta+$ $\left.\left.\left|a_{2}\right|\right) / \varepsilon_{2} K\right)(1+t)$ for some $\varepsilon_{2} \in(0,1)$, we obtain as previously that for the trajectories of the subsystem (5.18b) it holds that

$$
\begin{aligned}
\phi_{2}(s)|x(s)| & \leq|y(s)| \text { a.e for } s \in\left[t_{0}, t\right] \\
& \Rightarrow|y(t)| \leq \exp \left(-K\left(1-\varepsilon_{2}\right)\left(t-t_{0}\right)\right)\left|y_{0}\right| .
\end{aligned}
$$

It follows from (5.20a), (5.20b) that (5.14a), (5.14b) hold with $\phi_{i}(i=1,2)$ as previously defined, $\beta_{i}(t) \equiv 1(i=1,2)$, $\mu_{1}(t):=1-\varepsilon_{1}$ and $\mu_{2}(t):=K\left(1-\varepsilon_{2}\right)$. Finally, by using the elementary inequality

$$
\sup _{t \geq t_{0}}(1+t) \exp \left(-\left(1-\varepsilon_{1}\right) t\right) \leq \frac{3-2 \varepsilon_{1}}{1-\varepsilon_{1}} \exp \left(-\left(2-\varepsilon_{1}\right)\right)
$$

and taking into account definitions of $\beta_{i}, \phi_{i}, \mu_{i}(i=1,2)$, it follows that (5.15) holds and for $\varepsilon_{1}=1 / 2$ we have

$\gamma_{1}(t) \leq 2\left(\delta+\left|a_{1}\right|\right) \exp (-t) ; \gamma_{2}(t) \leq \frac{4\left(\delta+\left|a_{2}\right|\right)}{\varepsilon_{2} K} \exp \left(\frac{t-3}{2}\right)$

with $\gamma_{i}(i=1,2)$ as precisely defined by $(5.16 \mathrm{~b})$. Moreover, by using (5.21) and invoking our hypothesis (5.19), we can determine constants $\varepsilon_{2} \in(0,1)$ and $\delta>0$, in such a way that (5.16a) holds as well. Therefore, all hypotheses (5.14a), (5.14b), (5.15) and (5.16a), (5.16b) are fulfilled and Theorem 4.1 asserts that zero is nonuniformly in time GAS for system (5.18). 
Example 5.3: Consider the nonlinear planar system

$$
\dot{x}=-x^{3}+b_{1}(t) x y \quad \dot{y}=b_{2}(t) x^{2}-y+v .
$$

For the case $b_{1}(t) \equiv 1$ and $b_{2}(t) \equiv b_{2}$ (constant), the stability behavior of (5.22) is studied in [6] by applying the small-gain theorem of Jiang-Teel-Praly. It is proved that, if $\left|b_{2}\right|<1 / 2$, then the origin for the composite system (5.22) is uniformly in time GAS. Here, we consider the system (5.22) under the following more general hypothesis.

$H: b_{1}$ and $b_{2}: \Re^{+} \rightarrow \Re$ are $C^{0}$ functions and there exist a constant $K \geq 0$ and a positive nondecreasing function $\gamma \in$ $C^{1}\left(\mathcal{R}^{+} ;(0+\infty)\right)$ such that

$$
\begin{gathered}
r:=\sup _{t \geq 0} \frac{\dot{\gamma}(t)}{\gamma(t)}<1 \quad K<\frac{1}{2}(1-r) \\
\left|b_{1}(t)\right| \leq \gamma(t),\left|b_{2}(t)\right| \leq \frac{K}{\gamma(t)}, \quad \forall t \geq 0 .
\end{gathered}
$$

To simplify the analysis, we first invoke Remark 3.4, which asserts that nonuniform in time ISS remains invariant under input transformations T3, thus (5.22) satisfies the nonuniform in time ISS property from the input $v$, if and only if the following planar system satisfies the same property from the input $u$ :

$$
\begin{aligned}
& \dot{x}=-x^{3}+b_{1}(t) x y \\
& \dot{y}=b_{2}(t) x^{2}-y+\frac{c}{\gamma(t)} \operatorname{sgn}(u) u^{2}
\end{aligned}
$$

for arbitrary choice of $c>0$. Notice that subsystem (5.24a) with $y$ as input, satisfies the nonuniform in time ISS property and particularly, by virtue of first inequality of (5.23b), satisfies (4.1) with $\phi_{1}(t):=\gamma(t), \beta_{1}(t):=1, \rho_{1}(s):=\left(s / \varepsilon_{1}\right)^{1 / 2}$ and $\sigma_{1}(s, t):=s / \sqrt{1+2\left(1-\varepsilon_{1}\right) s^{2} t}$ for arbitrary choice of $\varepsilon_{1} \in(0,1)$. Indeed, by evaluating the time derivative $\dot{V}$ of the Lyapunov function $V(x)=x^{2} / 2$ along the trajectories of the subsystem (5.24a) with $\rho_{1}\left(\phi_{1}(t)|y|\right) \leq|x|$ and taking into account (5.23b) of our Hypothesis $\mathrm{H}$ and definitions of $\phi_{1}$ and $\rho_{1}$ we find

$\dot{V}=-x^{4}+b_{1}(t) x^{2} y \leq-x^{4}+\gamma(t) x^{2}|y| \leq-4\left(1-\varepsilon_{1}\right) V^{2}(x)$ provided that $\rho_{1}\left(\phi_{1}(t)|y|\right) \leq|x|$.
Therefore, by virtue of the previous property, equivalence of statements iii) and iv) of Proposition 2.5 and equivalence of statements ii) and iii) of Proposition 3.1 plus Remark 3.3, we deduce (4.1). Moreover, it may be shown that subsystem (5.24b) with $(x, u)$ as input satisfies the nonuniform in time ISS property and particularly, satisfies (4.2) with $\lambda:=1$, $\phi_{2}(t):=(1 / \gamma(t))^{1 / 2}, \beta_{2}(t):=1, \rho_{2}(s):=\left((K+c) / \varepsilon_{2}\right) s^{2}$ and $\sigma_{2}(s, t):=2 s \exp \left(-\left(1-\varepsilon_{2}\right) t\right)$ for arbitrary $\varepsilon_{2} \in(0,1)$. Indeed, the variation of constants formula for (5.24b) implies

$$
\begin{aligned}
|y(t)| \leq & \exp \left(-\left(t-t_{0}\right)\right)\left|y_{0}\right| \\
& \quad+\int_{t_{0}}^{t} \exp (-(t-\tau))\left(\left|b_{2}(\tau)\right| x^{2}(\tau)+c \frac{u^{2}(\tau)}{\gamma(\tau)}\right) d \tau
\end{aligned}
$$

Taking into account (5.23b) and the previous inequality, we obtain the equation shown at the bottom of the page, which implies (4.2). The definitions of $\lambda, \phi_{i}, \beta_{i}, \rho_{i}, \sigma_{i}(i=1,2)$ above, the fact that $\gamma$ is nondecreasing and inequalities (5.23a) guarantee that the following properties hold for appropriate selection of constants $0<\varepsilon_{1}, \varepsilon_{2}, L<1, c>0$ and for all $t_{0}, s \leq 0$ :

$$
\begin{aligned}
\lim _{t \rightarrow+\infty} \gamma(t) \exp \left(-\left(1-\varepsilon_{2}\right)\left(t-t_{0}\right)\right) & =0 \\
\lim _{t \rightarrow+\infty} \frac{K s^{2}}{\gamma(t)\left(1+2\left(1-\varepsilon_{1}\right) s^{2}\left(t-t_{0}\right)\right)} & =0
\end{aligned}
$$

$$
\begin{aligned}
(K+c) \sup _{t \geq t_{0}} \frac{\gamma(t)}{\gamma\left(t_{0}\right)} \exp \left(-\left(1-\varepsilon_{2}\right)\left(t-t_{0}\right)\right) & \leq \frac{1}{2} \varepsilon_{1} \varepsilon_{2} L^{2} \\
(K+c) \sup _{t \geq t_{0}} \frac{\gamma\left(t_{0}\right) s}{\gamma(t)\left(\varepsilon_{1}+2 s\left(1-\varepsilon_{1}\right) \gamma\left(t_{0}\right)\left(t-t_{0}\right)\right)} & \leq \frac{1}{2} \varepsilon_{2} L s .
\end{aligned}
$$

The first pair of conditions (5.25a) is equivalent to (4.3a), (4.3b) and the pair of inequalities (5.25b) is equivalent to (4.5a), (4.5b) with $a(s):=L s$. We conclude by Theorem 4.1 that (5.24) satisfies the nonuniform in time ISS property and thus, according to Remark 3.4, the same property is satisfied for system (5.22).

\section{APPENDIX}

Proof of Fact $V$ : Define $\beta(t, r) \quad:=$ $\sup \{|k(\tau, y)| ; 0 \leq \tau \leq t, y \in U \cap B[0, r]\} . \quad$ Obviously,

$$
\begin{aligned}
|y(t)| & \leq \max \left\{2 \exp \left(-\left(t-t_{0}\right)\right)\left|y_{0}\right|, 2(K+c) \int_{t_{0}}^{t} \frac{\exp (-(t-\tau))}{\gamma(\tau)}(\max \{|x(\tau)|,|u(\tau)|\})^{2} d \tau\right\} \\
& \leq \max \left\{2 \exp \left(-\left(1-\varepsilon_{2}\right)\left(t-t_{0}\right)\right)\left|y_{0}\right|, \sup _{t_{0} \leq \tau \leq t}\left(2(K+c) \frac{\exp \left(-\left(1-\varepsilon_{2}\right)(t-\tau)\right)}{\varepsilon_{2} \gamma(\tau)}(\max \{|x(\tau)|,|u(\tau)|\})^{2}\right)\right\} .
\end{aligned}
$$


$\beta$ satisfies all properties imposed in statement of Fact IV, hence, there exist functions $a \in K_{\infty}$ and $\phi \in K^{+}$such that $\beta(t, r) \leq a(\phi(t) r)$ for all $t, r \geq 0$. The latter in conjunction with definition of $\beta$ implies the desired (1.9).

Proof of Fact VI: Notice first that $\sup _{A} A\left(B\left(s, \tau-t_{0}\right), t-\tau\right) \leq \mu(s):=A(B(s, 0), 0)+s$ $t_{0} \leq \tau \leq t$

and $\mu$ is of class $K_{\infty}$. Define $g(s):=\sqrt{s}+s^{2}$, which is a $K_{\infty}$ function and let $\tilde{\rho}(t):=\sup \{A(B(s, \xi), t-\xi) / g(\mu(s)) ; s>0,0 \leq \xi \leq t\}$. It then follows that $0<\tilde{\rho}(\cdot) \leq 1$ and

$$
\begin{array}{r}
\sup _{t_{0} \leq \tau \leq t} A\left(B\left(s, \tau-t_{0}\right), t-\tau\right) \leq \tilde{\rho}\left(t-t_{0}\right) g(s) \\
\forall s \geq 0, \forall t \geq t_{0} .
\end{array}
$$

Clearly, for every $\varepsilon>0$ there exist positive numbers $0<$ $a(s)<b(s)$ such that

$$
x \in(0, a(\varepsilon)] \cup[b(\varepsilon),+\infty) \Rightarrow \frac{x}{\sqrt{x}+x^{2}} \leq \varepsilon .
$$

Thus, by recalling definitions of $\mu, g$ and using (A2), it follows that for every $\varepsilon>0$ it holds

$$
\begin{aligned}
& \mu(s) \in(0, a(\varepsilon)] \cup[b(\varepsilon),+\infty) \\
& \Rightarrow \sup \left\{\frac{A(B(s, \xi), t-\xi)}{g(\mu(s))} ; 0 \leq \xi \leq t\right\} \leq \varepsilon .
\end{aligned}
$$

For the case $\mu(s) \in(a(\varepsilon), b(\varepsilon))$, we obtain the estimate

$$
\begin{aligned}
\sup \left\{\frac{A(B(s, \xi), t-\xi)}{g(\mu(s))} ; 0 \leq \xi \leq t\right\} \\
\quad \leq \sup \left\{\frac{A(B(b(\varepsilon), \xi), t-\xi)}{g(a(\varepsilon))} ; 0 \leq \xi \leq t\right\} .
\end{aligned}
$$

Since $A, B \in K L$, it follows that for every $\varepsilon>0$ there exist $\xi_{1}(\varepsilon) \geq 0$ and $t_{1}(\varepsilon) \geq 0$ such that $\xi \geq \xi_{1}(\varepsilon) \Rightarrow A(B(b(\varepsilon), \xi), 0) \leq \varepsilon g(a(\varepsilon))$ and $t \geq t_{1}(\varepsilon) \Rightarrow A(B(b(\varepsilon), 0), t) \leq \varepsilon g(a(\varepsilon))$; consequently, we get

$$
\left.\begin{array}{c}
\xi \in\left[\xi_{1}(\varepsilon), t\right] \cup\left[0, t-t_{1}(\varepsilon)\right] \\
\mu(s) \in(a(\varepsilon), b(\varepsilon))
\end{array}\right\} \Rightarrow \frac{A(B(b(\varepsilon), \xi), t-\xi)}{g(a(\varepsilon))} \leq \varepsilon .
$$

Obviously, for $t \geq t_{1}(\varepsilon)+\xi_{1}(\varepsilon)$ we have $\left[\xi_{1}(\varepsilon), t\right] \cup[0, t-$ $\left.t_{1}(\varepsilon)\right]=[0, t]$, hence, from (A3)-(A5), and taking into account definition of $\tilde{\rho}$, we obtain $t \geq t_{1}(\varepsilon)+\xi_{1}(\varepsilon) \Rightarrow \tilde{\rho}(t) \leq \varepsilon$. Since $\varepsilon>0$ is arbitrary, we conclude that $\lim _{t \rightarrow+\infty} \tilde{\rho}(t)=0$. Let $\rho: \Re^{+} \rightarrow(0,+\infty)$ be a $C^{0}$ strictly decreasing function with $\lim _{t \rightarrow+\infty} \rho(t)=0$ such that $\tilde{\rho}(t) \leq \rho(t)$ for all $t \geq 0$ and define $R(s, t):=\rho(t) g(\mu(s))$. Obviously, $R \in K L$ and the desired (1.10) is a consequence of (A1).

Proof of Fact VII: First, it can be easily shown that property $\lim _{t \rightarrow+\infty} \phi_{2}(t) \rho\left(\phi_{1}(t) \sigma\left(s, t-t_{0}\right)\right)=0$ is equivalent to the following one.

$(P)$ : "For any $\varepsilon>0, T \geq 0, R \geq 0$, there exists a $\tau=$ $\tau(\varepsilon, T, R) \geq 0$, such that $\phi_{2}(t) \rho\left(\phi_{1}(t) \sigma\left(s, t-t_{0}\right)\right) \leq \varepsilon$ for all $t-T \geq \tau, t_{0} \in[0, T]$ and $s \leq R . "$

Next, define $\mu(s):=\sigma(s, \overline{0})+s$ and $v(s):=\rho(s)+s$. Moreover, define for $\varepsilon>0, T \geq 0 r_{i}(T, \varepsilon):=$ $\sup \left\{\phi_{i}(\tau) ; 0 \leq \tau \leq T+\tau(\varepsilon, T, \varepsilon)\right\}, i=1,2$ and notice that property $(\mathrm{P})$ implies

$$
\begin{aligned}
& \sup _{0 \leq t_{0} \leq T} \sup _{t \geq t_{0}} \phi_{2}(t) \rho\left(\phi_{1}(t) \sigma\left(s, t-t_{0}\right)\right) \leq \varepsilon \\
& \forall s \leq \delta(T, \varepsilon):=\min \left\{\varepsilon, \mu^{-1}\left(\frac{1}{r_{1}(T, \varepsilon)} v^{-1}\left(\frac{\varepsilon}{r_{2}(T, \varepsilon)}\right)\right)\right\} .
\end{aligned}
$$

Also, define: $a(T, s):=\sup _{0 \leq t_{0} \leq T} \sup _{t \geq t_{0}} \phi_{2}(t) \rho\left(\phi_{1}(t) \sigma\left(s, t-t_{0}\right)\right)$ and notice that $a$ is finite-valued, since by virtue of property (P) and definitions of $r_{i}$ we have the equation at the bottom of the page. Furthermore, (A6) implies $\lim _{s \rightarrow 0^{+}} a(T, s)=0=$ $a(T, 0)$ for all $T \geq 0$ and, according to definition of $a$ above, it follows that for each $T$ and $s$ the mappings $a(T, \cdot), a(\cdot, s)$ are both nondecreasing. Thus, $a$ satisfies all requirements of Fact IV and consequently, there exist functions $A \in K_{\infty}$ and $\theta \in K^{+}$ with $a\left(t_{0}, s\right) \leq A\left(\theta\left(t_{0}\right) s\right)$, for all $t_{0}, s \geq 0$. It turns out that

$$
\phi_{2}(t) \rho\left(\phi_{1}(t) \sigma\left(s, t-t_{0}\right)\right) \leq A\left(\theta\left(t_{0}\right) s\right) \quad \forall t \geq t_{0} .
$$

Without loss of generality we may assume that $\theta \in K^{+}$is nondecreasing. Let $p \in K^{+}$be any nondecreasing function with $\lim _{t \rightarrow+\infty} p(t)=+\infty, p(t) \geq 1$ for all $t \geq 0$. Define $\lambda(s):=\sqrt{s}+s^{2}$ and

$$
\omega(t):=\sup \left\{\frac{\phi_{2}\left(t_{0}+t\right) \rho\left(\phi_{1}\left(t_{0}+t\right) \sigma(s, t)\right)}{p\left(t_{0}\right) \lambda\left(A\left(\theta\left(t_{0}\right) s\right)\right)}, s>0, t_{0} \geq 0\right\} .
$$

Clearly, definition (A8) in conjunction with (A7) implies that $0 \leq \omega(t) \leq 1$ for all $t \geq 0$. Furthermore, according to definition (A) $)$, we have

$$
\begin{aligned}
\phi_{2}(t) \rho\left(\phi_{1}(t) \sigma\left(s, t-t_{0}\right)\right) & \leq \omega\left(t-t_{0}\right) p\left(t_{0}\right) \lambda\left(A\left(\theta\left(t_{0}\right) s\right)\right) \\
\forall t & \geq t_{0}, s \geq 0, t_{0} \geq 0 .
\end{aligned}
$$

$$
\begin{aligned}
\sup _{t \geq t_{0}} \phi_{2}(t) \rho\left(\phi_{1}(t) \sigma\left(s, t-t_{0}\right)\right) & \leq \max \left\{\begin{array}{l}
\left.\sup _{t_{0} \leq t \leq T+\tau(s, T, s)} \phi_{2}(t) \rho\left(\phi_{1}(t) \sigma\left(s, t-t_{0}\right)\right), \sup _{t \geq T+\tau(s, T, s)} \phi_{2}(t) \rho\left(\phi_{1}(t) \sigma\left(s, t-t_{0}\right)\right)\right\} \\
\leq \max \left\{\sup _{t_{0} \leq t \leq T+\tau(s, T, s)} \phi_{2}(t) \rho\left(\phi_{1}(t) \sigma(s, 0)\right), s\right\} \leq r_{2}(T, s) \rho\left(r_{1}(T, s) \sigma(s, 0)\right)+s<+\infty
\end{array}\right.
\end{aligned}
$$

$\forall s>0, T \geq 0$, and $t_{0} \in[0, T]$. 
By recalling Fact $\mathrm{V}$, there exists a $K_{\infty}$ function $\zeta$ and a function $B$ of class $K^{+}$such that

$$
p(t) \lambda(A(\theta(t) s)) \leq \zeta(B(t) s) \quad \forall(t, s) \in\left(\Re^{+}\right)^{2} .
$$

\section{Claim: $\lim _{t \rightarrow+\infty} \omega(t)=0$.}

Proof of Claim: Let $\varepsilon>0$ be arbitrary. Clearly, for every $\varepsilon>0$ there exist positive numbers $0<a(\varepsilon)<b(\varepsilon)$ such that implication (A2) holds. Moreover, there exists some finite time $t_{1}:=t_{1}(\varepsilon) \geq 0$ such that $t \in\left[t_{1}(\varepsilon),+\infty\right) \Rightarrow p(t) \geq 1 / \varepsilon$. The latter, in conjunction with (A2) and (A7), implies

$$
\begin{aligned}
& \left.\begin{array}{c}
A\left(\theta\left(t_{0}\right) s\right) \in(0, a(\varepsilon)] \cup[b(\varepsilon),+\infty) \\
\text { or } \\
t_{0} \geq t_{1}(\varepsilon)
\end{array}\right\} \Rightarrow \\
& \frac{\phi_{2}\left(t_{0}+t\right) \rho\left(\phi_{1}\left(t_{0}+t\right) \sigma(s, t)\right)}{p\left(t_{0}\right) \lambda\left(A\left(\theta\left(t_{0}\right) s\right)\right)} \leq \varepsilon .
\end{aligned}
$$

We finally examine the case $A\left(\theta\left(t_{0}\right) s\right) \in[a(\varepsilon), b(\varepsilon)]$ and $t_{0} \in\left[0, t_{1}(\varepsilon)\right]$. In this case we invoke property $(\mathrm{P})$, which asserts existence of a constant $\tau(\varepsilon) \geq 0$ such that $\phi_{2}\left(t_{0}+t\right) \rho\left(\phi_{1}\left(t_{0}+t\right) \sigma(s, t)\right) \leq \varepsilon r(\varepsilon)$ for all $t \geq \tau(\varepsilon)$, $t_{0} \in\left[0, t_{1}(\varepsilon)\right]$ and $s \leq(1 / \theta(0)) A^{-1}(b(\varepsilon))$, where $r(\varepsilon):=p(0) \lambda(A(\theta(0) a(\varepsilon)))$. Thus, for $t \geq \tau(\varepsilon)$ we obtain

$$
\frac{\phi_{2}\left(t_{0}+t\right) \rho\left(\phi_{1}\left(t_{0}+t\right) \sigma(s, t)\right)}{p\left(t_{0}\right) \lambda\left(A\left(\theta\left(t_{0}\right) s\right)\right)} \leq \frac{\phi_{2}\left(t_{0}+t\right) \rho\left(\phi_{1}\left(t_{0}+t\right) \sigma(s, t)\right)}{r(\varepsilon)} \leq \varepsilon .
$$

This inequality, in conjunction with (A11) and definition (A8), implies that for all $t \geq \tau(\varepsilon)$ we have $\omega(t) \leq \varepsilon$ and, since $\varepsilon>0$ is arbitrary, we conclude that $\lim _{t \rightarrow+\infty} \omega(t)=0$. Finally, let $\tilde{\omega}: \Re^{+} \rightarrow(0,+\infty)$ be a continuous and strictly decreasing function with $\lim _{t \rightarrow+\infty} \tilde{\omega}(t)=0$ and $\omega(t) \leq \tilde{\omega}(t)$ for all $t \geq 0$. The latter, in conjunction with (A9) and (A10) asserts that the desired inequality (1.11) holds with $S(s, t):=\tilde{\omega}(t) \zeta(s)$.

Proof of Lemma 2.8: Since $0 \in \Re^{n}$ is nonuniformly in time GAS for the unforced system $\dot{x}=f(t, x, 0)$ it follows, by the converse Lyapunov theorem in [11], that there exist functions $V \in C^{\infty}\left(\Re^{+} \times \Re^{n} ; \Re^{+}\right), a_{1}, a_{2} \in K_{\infty}$ and $p \in K^{+}$, such that (2.5a) holds and

$$
\begin{aligned}
\frac{\partial V}{\partial t}(t, x)+\frac{\partial V}{\partial x}(t, x) f(t, x, 0) & \leq-V(t, x) \\
\forall(t, x) & \in \Re^{+} \times \Re^{n} .
\end{aligned}
$$

Define:

$$
\begin{aligned}
& k_{1}(t, s, u):=\sup _{|x| \leq s, 0 \leq \tau \leq t}\left|\frac{\partial V}{\partial x}(\tau, x)(f(\tau, x, u)-f(\tau, x, 0))\right| \\
& k_{2}(t, s, x):=\sup _{|u| \leq s, 0 \leq \tau \leq t}\left|\frac{\partial V}{\partial x}(\tau, x)(f(\tau, x, u)-f(\tau, x, 0))\right| .
\end{aligned}
$$

Then we may use Fact $\mathrm{V}$, to determine functions $\zeta \in K_{\infty}$ and $\beta \in K^{+}$such that $k_{1}(t, t, u) \leq \zeta(\beta(t)|u|) ; k_{2}(t, t, x) \leq$ $\zeta(\beta(t)|x|)$. Without loss of generality we may assume that $\beta \in$
$K^{+}$is strictly increasing and satisfies $\beta(t) \geq 1$, for all $t \geq 0$. It then follows from (A13) and previous inequalities that

$$
\begin{aligned}
& \frac{\partial V}{\partial x}(t, x)(f(t, x, u)-f(t, x, 0)) \\
& \leq \min \{\zeta(\beta(t+|x|)|u|), \zeta(\beta(t+|u|)|x|)\} \\
& \forall(t, x, u) \in \Re^{+} \times \Re^{n} \times \Re^{m} .
\end{aligned}
$$

Using the inequality $\beta(t+s) \leq \beta(2 t)+\beta(2 s)$ and defining $\tilde{\beta}(t):=\beta(2 t), \gamma(r, s):=\min \{\beta(2 r) s+s, \beta(2 s) r+r\}$ we obtain from (A14):

$$
\begin{aligned}
\frac{\partial V}{\partial x}(t, x)(f(t, x, u)-f(t, x, 0)) & \leq \zeta(\tilde{\beta}(t) \gamma(|x|,|u|)) \\
\forall(t, x, u) & \left.\in \Re^{+} \times \Re^{n} \times \Re^{m} . \quad\right)
\end{aligned}
$$

By recalling Facts I and II, there exist functions $\sigma \in K_{\infty}$, $\tilde{\zeta} \in K_{\infty}$ such that $\gamma(r, s) \leq \sigma(r) \sigma(s)$ and $\zeta(r s) \leq \tilde{\zeta}(r) \tilde{\zeta}(s)$, thus (A15) implies that the following inequality holds for all $(t, x, u) \in \Re^{+} \times \Re^{n} \times \Re^{m}:$

$$
\begin{aligned}
& \frac{\partial V}{\partial x}(t, x)(f(t, x, u)-f(t, x, 0)) \\
\leq & \tilde{\zeta}(\sigma(|x|)) \tilde{\zeta}(\tilde{\beta}(t) \sigma(|u|)) \\
\leq & \exp (-2 t) \rho\left(\mu(t) \frac{|x|}{\mu(t)}\right) \exp (2 t) \tilde{\zeta}(\tilde{\beta}(t) \sigma(|u|))
\end{aligned}
$$

where $\rho(s):=\tilde{\zeta}(\sigma(s))$. We again recall Facts II and $\mathrm{V}$ to determine functions $a_{3} \in K_{\infty}, \kappa \in K^{+}$ and $a_{4} \in K_{\infty}$ such that $\rho(r s) \leq a_{3}(r) a_{3}(s)$ and $\exp (2 t) a_{3}(\mu(t)) \tilde{\zeta}(\tilde{\beta}(t) \sigma(s)) \leq a_{4}(\kappa(t) s)$. The desired (2.5b) is then an immediate consequence of (A16). The proof is complete.

Proof of Claim Made in the Proof of Theorem 4.1: We proceed by first establishing the following properties.

Property 1: Under Hypothesis A3 of Theorem 4.1, there exist functions $B_{i} \in K L$ and $\tilde{\beta}_{i} \in K^{+}(i=1,2)$ such that for all $s \geq 0$ and $t \geq t_{0}$ and for every nonnegative $s_{1}, s_{2}$ with $s_{1}, s_{2} \leq s$ the following inequalities hold:

$$
\begin{aligned}
\sigma_{1} & \left(\beta_{1}\left(t_{0}\right) s_{1}, t-t_{0}\right) \\
& +\sup _{t_{0} \leq \tau \leq t} \sigma_{1}\left(\beta_{1}(\tau) \rho_{1}\left(\phi_{1}(\tau) \sigma_{2}\left(\beta_{2}\left(t_{0}\right) s_{2}, \tau-t_{0}\right)\right), t-\tau\right) \\
\leq & B_{1}\left(\tilde{\beta}_{1}\left(t_{0}\right) s, t-t_{0}\right) \\
\sigma_{2} & \left(\beta_{2}\left(t_{0}\right) s_{1}, t-t_{0}\right) \\
& +\sup _{t_{0} \leq \tau \leq t} \sigma_{2}\left(\beta_{2}(\tau) \rho_{2}\left(\phi_{2}(\tau) \lambda \sigma_{1}\left(\beta_{1}\left(t_{0}\right) s_{2}, \tau-t_{0}\right)\right), t-\tau\right) \\
\leq & B_{2}\left(\tilde{\beta}_{2}\left(t_{0}\right) s, t-t_{0}\right) .
\end{aligned}
$$

Proof: By using Fact VII and Hypothesis A3, there exist functions $S_{i} \in K L$ and $\delta_{i} \in K^{+}(i=1,2)$ such that

$$
\beta_{1}(t) \rho_{1}\left(\phi_{1}(t) \sigma_{2}\left(s, t-t_{0}\right)\right) \leq S_{1}\left(\delta_{1}\left(t_{0}\right) s, t-t_{0}\right) \forall t \geq t_{0} s \geq 0
$$

$\beta_{2}(t) \rho_{2}\left(\phi_{2}(t) \lambda \sigma_{1}\left(s, t-t_{0}\right)\right) \leq S_{2}\left(\delta_{2}\left(t_{0}\right) s, t-t_{0}\right) \forall t \geq t_{0} s \geq 0$. 
Furthermore, by using Fact VI, there exist functions $R_{i} \in$ $K L(i=1,2)$ such that

$$
\begin{aligned}
& \sup _{t_{0} \leq \tau \leq t} \sigma_{1}\left(S_{1}\left(s, \tau-t_{0}\right), t-\tau\right) \leq R_{1}\left(s, t-t_{0}\right) \\
& \sup _{t_{0} \leq \tau \leq t} \sigma_{2}\left(S_{2}\left(s, \tau-t_{0}\right), t-\tau\right) \leq R_{2}\left(s, t-t_{0}\right)
\end{aligned}
$$$$
\forall t \geq t_{0} s \geq 0
$$

We define $\tilde{\beta}_{1}(t):=\delta_{1}(t) \beta_{2}(t)+\beta_{1}(t)$ and $\tilde{\beta}_{2}(t):=$ $\delta_{2}(t) \beta_{1}(t)+\beta_{2}(t)$. Inequalities (A19)-(A22), along with previous definitions, imply that for all $s \geq 0$ and $t \geq t_{0}$ the following inequalities hold:

$$
\begin{aligned}
\sup _{t_{0} \leq \tau \leq t} & \sigma_{1}\left(\beta_{1}(\tau) \rho_{1}\left(\phi_{1}(\tau) \sigma_{2}\left(\beta_{2}\left(t_{0}\right) s, \tau-t_{0}\right)\right), t-\tau\right) \\
& \leq R_{1}\left(\tilde{\beta}_{1}\left(t_{0}\right) s, t-t_{0}\right) \\
\sup _{t_{0} \leq \tau \leq t} & \sigma_{2}\left(\beta_{2}(\tau) \rho_{2}\left(\phi_{2}(\tau) \lambda \sigma_{1}\left(\beta_{1}\left(t_{0}\right) s, \tau-t_{0}\right)\right), t-\tau\right) \\
& \leq R_{2}\left(\tilde{\beta}_{2}\left(t_{0}\right) s, t-t_{0}\right) .
\end{aligned}
$$

We define for $i=1,2$, the following functions of class $K L$ : $B_{i}(s, t):=R_{i}(s, t)+\sigma_{i}(s, t)$. The previous definitions, in conjunction with inequalities (A23) and (A24), imply the desired (A17) and (A18).

A consequence of Property 1 and Hypothesis A3 of Theorem 4.1 is the following.
Property 2: Under Hypothesis A3 of Theorem 4.1, there exist $\zeta_{i} \in K_{\infty}$ and $\tilde{\delta}_{i} \in K^{+}(i=1,2)$, such that for every $u \in L_{\mathrm{loc}}^{\infty}\left(\left[t_{0},+\infty\right)\right)$, the inequalities shown in (A25)-(A28) at the bottom of the page, hold for all $t \geq t_{0}$.

Proof: Fact V guarantees existence of functions $\zeta_{i} \in K_{\infty}$ and $\tilde{\delta}_{i} \in K^{+}(i=1,2)$ such that for all $t \geq 0$ and the following inequalities hold:

$$
\begin{aligned}
& 2 \sigma_{1}\left(\beta_{1}(t) \rho_{1}\left(\phi_{1}(t) s\right), 0\right)+2 \sigma_{1}\left(S_{1}\left(\delta_{1}(t) \beta_{2}(t) \rho_{2}\left(\phi_{2}(t) s\right), 0\right), 0\right) \\
& \leq \zeta_{1}\left(\tilde{\delta}_{1}(t) s\right) \\
& 2 \sigma_{2}\left(\beta_{2}(t) \rho_{2}\left(\phi_{2}(t) s\right), 0\right)+2 \sigma_{2}\left(S_{2}\left(\delta_{2}(t) \beta_{1}(t) \rho_{1}\left(\phi_{1}(t) s\right), 0\right), 0\right) \\
& \leq \zeta_{2}\left(\tilde{\delta}_{2}(t) s\right)
\end{aligned}
$$

where $S_{i} \in K L$ and $\delta_{i} \in K^{+}(i=1,2)$ are defined in (A19) and (A20). Moreover, by (A19) and (A20), we obtain the equation, shown at the bottom of the page. These inequalities, in conjunction with (A29) and (A30), assert that the desired (A25), (A26), (A27), and (A28) hold for appropriate $\zeta_{i} \in K_{\infty}$ and $\tilde{\delta}_{i} \in K^{+}(i=1,2)$.

A straightforward consequence of Hypothesis A4 of Theorem 4.1 is the following property.

Property 3: Under Hypotheses A3 and A4 of Theorem 4.1, for every pair of mappings $x(\cdot):\left[t_{0},+\infty\right) \rightarrow \Re^{n}, y(\cdot)$ : $\left[t_{0},+\infty\right) \rightarrow \Re^{k}$ of class $C^{0}\left(\left[t_{0},+\infty\right)\right)$, the following inequalities hold for all $t \geq t_{0}$; see (A31) and (A32), shown at the bottom of the page, where $a$ is the function involved in (4.4) and (4.5a), (4.5b).

$$
\begin{aligned}
\sup _{t_{0} \leq \tau \leq t} \sigma_{1}\left(\beta_{1}(\tau) \rho_{1}\left(\phi_{1}(\tau) \sup _{t_{0} \leq \xi \leq \tau} \sigma_{2}\left(\beta_{2}(\xi) \rho_{2}\left(\phi_{2}(\xi)|u(\xi)|\right), \tau-\xi\right)\right), t-\tau\right) & \leq \frac{1}{2} \sup _{t_{0} \leq \tau \leq t} \zeta_{1}\left(\tilde{\delta}_{1}(\tau)|u(\tau)|\right) \\
\sup _{t_{0} \leq \tau \leq t} \sigma_{2}\left(\beta_{2}(\tau) \rho_{2}\left(\lambda \phi_{2}(\tau) \sup _{t_{0} \leq \xi \leq \tau} \sigma_{1}\left(\beta_{1}(\xi) \rho_{1}\left(\phi_{1}(\xi)|u(\xi)|\right), \tau-\xi\right)\right), t-\tau\right) & \leq \frac{1}{2} \sup _{t_{0} \leq \tau \leq t} \zeta_{2}\left(\tilde{\delta}_{2}(\tau)|u(\tau)|\right) \\
\sup _{t_{0} \leq \tau \leq t} \sigma_{1}\left(\beta_{1}(\tau) \rho_{1}\left(\phi_{1}(\tau)|u(\tau)|\right), t-\tau\right) & \leq \frac{1}{2} \sup _{t_{0} \leq \tau \leq t} \zeta_{1}\left(\tilde{\delta}_{1}(\tau)|u(\tau)|\right) \\
\sup _{t_{0} \leq \tau \leq t} \sigma_{2}\left(\beta_{2}(\tau) \rho_{2}\left(\phi_{2}(\tau)|u(\tau)|\right), t-\tau\right) & \leq \frac{1}{2} \sup _{t_{0} \leq \tau \leq t} \zeta_{2}\left(\tilde{\delta}_{2}(\tau)|u(\tau)|\right) .
\end{aligned}
$$

$$
\begin{aligned}
\sup _{t_{0} \leq \tau \leq t} & \sigma_{1}\left(\beta_{1}(\tau) \rho_{1}\left(\phi_{1}(\tau) \sup _{t_{0} \leq \xi \leq \tau} \sigma_{2}\left(\beta_{2}(\xi) \rho_{2}\left(\phi_{2}(\xi)|u(\xi)|\right), \tau-\xi\right)\right), t-\tau\right) \\
& \leq \sup _{t_{0} \leq \tau \leq t} \sup _{t_{0} \leq \xi \leq \tau} \sigma_{1}\left(\beta_{1}(\tau) \rho_{1}\left(\phi_{1}(\tau) \sigma_{2}\left(\beta_{2}(\xi) \rho_{2}\left(\phi_{2}(\xi)|u(\xi)|\right), \tau-\xi\right)\right), 0\right) \\
& \leq \sup _{t_{0} \leq \tau \leq t} \sup _{t_{0} \leq \xi \leq \tau} \sigma_{1}\left(S_{1}\left(\delta_{1}(\xi) \beta_{2}(\xi) \rho_{2}\left(\phi_{2}(\xi)|u(\xi)|\right), \tau-\xi\right), 0\right) \\
\sup _{t_{0} \leq \tau \leq t} & \sigma_{2}\left(\beta_{2}(\tau) \rho_{2}\left(\lambda \phi_{2}(\tau) \sup _{t_{0} \leq \xi \leq \tau} \sigma_{1}\left(\beta_{1}(\xi) \rho_{1}\left(\phi_{1}(\xi)|u(\xi)|\right), \tau-\xi\right)\right), t-\tau\right) \\
& \leq \sup _{t_{0} \leq \tau \leq t} \sup _{t_{0} \leq \xi \leq \tau} \sigma_{2}\left(\beta_{2}(\tau) \rho_{2}\left(\lambda \phi_{2}(\tau) \sigma_{1}\left(\beta_{1}(\xi) \rho_{1}\left(\phi_{1}(\xi)|u(\xi)|\right), \tau-\xi\right)\right), 0\right) \\
& \leq \sup _{t_{0} \leq \tau \leq t} \sup _{t_{0} \leq \xi \leq \tau} \sigma_{2}\left(S_{2}\left(\delta_{2}(\xi) \beta_{1}(\xi) \rho_{1}\left(\phi_{1}(\xi)|u(\xi)|\right), \tau-\xi\right), 0\right) \quad \forall t \geq t_{0} .
\end{aligned}
$$



(4.5b).

Proof: Immediate consequence of inequalities (4.5a),

We are now in a position to prove our Claim. Clearly, for every $u \in L_{\mathrm{loc}}^{\infty}\left(\left[t_{0},+\infty\right)\right)$, there exists a maximal interval $\left[t_{0}, T\right)$ with $t_{0}<T \leq+\infty$ such that the corresponding solution of (1.4) exists. Exploiting (4.1) and (4.2), we obtain the following estimates for the components $x(\cdot)$ and $y(\cdot)$ of the solution $\tilde{x}(\cdot)$ of $(1.4)$ on the interval $\left[t_{0}, T\right)$

$$
\begin{aligned}
|x(t)| & \leq \max \left\{a_{1}, a_{2}, a_{3}\right\} \\
a_{1} & :=\sigma_{1}\left(\beta_{1}\left(t_{0}\right)\left|x_{0}\right|, t-t_{0}\right) \\
a_{2} & :=\sup _{t_{0} \leq \tau \leq t} \sigma_{1}\left(\beta_{1}(\tau) \rho_{1}\left(\phi_{1}(\tau)|y(\tau)|\right), t-\tau\right) \\
a_{3} & :=\sup _{t_{0} \leq \tau \leq t} \sigma_{1}\left(\beta_{1}(\tau) \rho_{1}\left(\phi_{1}(\tau)|u(\tau)|\right), t-\tau\right)
\end{aligned}
$$

$$
\begin{aligned}
|y(t)| & \leq \max \left\{b_{1}, b_{2}, b_{3}\right\} ; \\
b_{1} & :=\sigma_{2}\left(\beta_{2}\left(t_{0}\right)\left|y_{0}\right|, t-t_{0}\right) \\
b_{2} & :=\sup _{t_{0} \leq \tau \leq t} \sigma_{2}\left(\beta_{2}(\tau) \rho_{2}\left(\phi_{2}(\tau) \lambda|x(\tau)|\right), t-\tau\right) \\
b_{3} & :=\sup _{t_{0} \leq \tau \leq t} \sigma_{2}\left(\beta_{2}(\tau) \rho_{2}\left(\phi_{2}(\tau)|u(\tau)|\right), t-\tau\right) .
\end{aligned}
$$

Combining (A33) and (A34), it follows that (A35)-(A36), as shown at the bottom of the page, hold. Using (A17), (A18), (A25)-(A28), and (A31)-(A36), we obtain the estimates, shown in (A37)-(A38) at the bottom of the page, on the interval $\left[t_{0}, T\right)$. From (A37) and (A38), (A39)-(A40), as shown at the top of the next page, hold. Inequalities (A39) and (A40), in conjunction with our Hypothesis (4.4), imply that (A41)-(A42), as shown at the top of the next page, hold.It follows from (A41), (A42) that the solution $\tilde{x}(\cdot)$ does not have a finite escape time, equivalently

$$
\begin{aligned}
& \sup _{t_{0} \leq \tau \leq t} \sigma_{1}\left(\beta_{1}(\tau) \rho_{1}\left(\phi_{1}(\tau) \sup _{t_{0} \leq \xi \leq \tau} \sigma_{2}\left(\beta_{2}(\xi) \rho_{2}\left(\phi_{2}(\xi) \lambda|x(\xi)|\right), \tau-\xi\right)\right), t-\tau\right) \leq a\left(\sup _{t_{0} \leq \tau \leq t}|x(\tau)|\right) \\
& \sup _{t_{0} \leq \tau \leq t} \sigma_{2}\left(\beta_{2}(\tau) \rho_{2}\left(\lambda \phi_{2}(\tau) \sup _{t_{0} \leq \xi \leq \tau} \sigma_{1}\left(\beta_{1}(\xi) \rho_{1}\left(\phi_{1}(\xi)|y(\xi)|\right), \tau-\xi\right)\right), t-\tau\right) \leq a\left(\sup _{t_{0} \leq \tau \leq t}|y(\tau)|\right)
\end{aligned}
$$

$$
\begin{aligned}
&|x(t)| \leq \max _{2}\left\{a_{1}, a_{2} b_{1}, a_{2} b_{2}, a_{2} b_{3}, a_{3}\right\} \\
& a_{2} b_{1}:=\sup _{t_{0} \leq \tau \leq t} \sigma_{1}\left(\beta_{1}(\tau) \rho_{1}\left(\phi_{1}(\tau) \sigma_{2}\left(\beta_{2}\left(t_{0}\right)\left|y_{0}\right|, \tau-t_{0}\right)\right), t-\tau\right) \\
& a_{2} b_{2}:=\sup _{t_{0} \leq \tau \leq t} \sigma_{1}\left(\beta_{1}(\tau) \rho_{1}\left(\phi_{1}(\tau) \sup _{t_{0} \leq \xi \leq \tau} \sigma_{2}\left(\beta_{2}(\xi) \rho_{2}\left(\phi_{2}(\xi) \lambda|x(\xi)|\right), \tau-\xi\right)\right), t-\tau\right) \\
& a_{2} b_{3}:=\sup _{t_{0} \leq \tau \leq t} \sigma_{1}\left(\beta_{1}(\tau) \rho_{1}\left(\phi_{1}(\tau) \sup _{t_{0} \leq \xi \leq \tau} \sigma_{2}\left(\beta_{2}(\xi) \rho_{2}\left(\phi_{2}(\xi)|u(\xi)|\right), \tau-\xi\right)\right), t-\tau\right) \\
&|y(t)| \leq \max _{2}\left\{b_{1}, b_{2} a_{1}, b_{2} a_{2}, b_{2} a_{3}, b_{3}\right\} \\
& b_{2} a_{1}:=\sup _{t_{0} \leq \tau \leq t} \sigma_{2}\left(\beta_{2}(\tau) \rho_{2}\left(\phi_{2}(\tau) \lambda \sigma_{1}\left(\beta_{1}\left(t_{0}\right)\left|x_{0}\right|, \tau-t_{0}\right)\right), t-\tau\right) \\
& b_{2} a_{2}:=\sup _{t_{0} \leq \tau \leq t} \sigma_{2}\left(\beta_{2}(\tau) \rho_{2}\left(\lambda \phi_{2}(\tau) \sup _{t_{0} \leq \xi \leq \tau} \sigma_{1}\left(\beta_{1}(\xi) \rho_{1}\left(\phi_{1}(\xi)|y(\xi)|\right), \tau-\xi\right)\right), t-\tau\right) \\
& b_{2} a_{3}:=\sup _{t_{0} \leq \tau \leq t} \sigma_{2}\left(\beta_{2}(\tau) \rho_{2}\left(\lambda \phi_{2}(\tau) \sup _{t_{0} \leq \xi \leq \tau} \sigma_{1}\left(\beta_{1}(\xi) \rho_{1}\left(\phi_{1}(\xi)|u(\xi)|\right), \tau-\xi\right)\right), t-\tau\right) .
\end{aligned}
$$

$$
\begin{aligned}
& |x(t)| \leq \max \left\{B_{1}\left(\tilde{\beta}_{1}\left(t_{0}\right)\left|\tilde{x}_{0}\right|, t-t_{0}\right), a\left(\sup _{t_{0} \leq \tau \leq t}|x(\tau)|\right), \sup _{t_{0} \leq \tau \leq t} \zeta_{1}\left(\tilde{\delta}_{1}(\tau)|u(\tau)|\right)\right\} \\
& |y(t)| \leq \max \left\{B_{2}\left(\tilde{\beta}_{2}\left(t_{0}\right)\left|\tilde{x}_{0}\right|, t-t_{0}\right), a\left(\sup _{t_{0} \leq \tau \leq t}|y(\tau)|\right), \sup _{t_{0} \leq \tau \leq t} \zeta_{2}\left(\tilde{\delta}_{2}(\tau)|u(\tau)|\right)\right\} .
\end{aligned}
$$




$$
\begin{aligned}
& \sup _{t_{0} \leq \tau \leq t}|x(\tau)| \leq \max \left\{B_{1}\left(\tilde{\beta}_{1}\left(t_{0}\right)\left|\tilde{x}_{0}\right|, 0\right), a\left(\sup _{t_{0} \leq \tau \leq t}|x(\tau)|\right), \sup _{t_{0} \leq \tau \leq t} \zeta_{1}\left(\tilde{\delta}_{1}(\tau)|u(\tau)|\right)\right\} \\
& \sup _{t_{0} \leq \tau \leq t}|y(\tau)| \leq \max \left\{B_{2}\left(\tilde{\beta}_{2}\left(t_{0}\right)\left|\tilde{x}_{0}\right|, 0\right), a\left(\sup _{t_{0} \leq \tau \leq t}|y(\tau)|\right), \sup _{t_{0} \leq \tau \leq t} \zeta_{2}\left(\tilde{\delta}_{2}(\tau)|u(\tau)|\right)\right\} .
\end{aligned}
$$

$$
\begin{aligned}
& \sup _{t_{0} \leq \tau<t}|x(\tau)| \leq \max \left\{B_{1}\left(\tilde{\beta}_{1}\left(t_{0}\right)\left|\tilde{x}_{0}\right|, 0\right), \sup _{t_{0} \leq \tau \leq t} \zeta_{1}\left(\tilde{\delta}_{1}(\tau)|u(\tau)|\right)\right\} \\
& \sup _{t_{0} \leq \tau<t}|y(\tau)| \leq \max \left\{B_{2}\left(\tilde{\beta}_{2}\left(t_{0}\right)\left|\tilde{x}_{0}\right|, 0\right), \sup _{t_{0} \leq \tau \leq t} \zeta_{2}\left(\tilde{\delta}_{2}(\tau)|u(\tau)|\right)\right\} .
\end{aligned}
$$

$T=+\infty$. Furthermore, this implies that inequalities (A37), (A38), (A41), and (A42) hold for all $t \geq t_{0}$, thus (4.7a),(4.7b) and $(4.8 \mathrm{a}),(4.8 \mathrm{~b})$ are established.

\section{ACKNOWLEDGMENT}

The authors would like to thank Prof. E.D. Sontag who kindly accepted to present part of this work at the conference of MTNS 2002.

\section{REFERENCES}

[1] D. Angeli and E. D. Sontag, "Forward completeness, unbounded observability and their Lyapunov characterizations," Syst. Control Lett., vol. 38, no. 4-5, pp. 209-217, 1999.

[2] D. Angeli, E. D. Sontag, and Y. Wang, "A characterization of integral input-to-State stability," IEEE Trans. Automat. Contr., vol. 45, pp. 1082-1096, Aug. 2000.

[3] Z. Artstein, "Stabilization with relaxed controls," Nonlinear Anal., vol. TMA 7, pp. 1163-1173, 1983.

[4] A. Bloch, P. Crouch, J. Baillieul, and J. Marsden, Nonholonomic Mechanics and Control. New York: Springer-Verlag, 2003.

[5] L. Grune, Asymptotic Behavior of Dynamical and Control Systems Under Perturbation and Discretization, ser. Lecture Notes in Mathematics, vol. 1783, 2002, to be published.

[6] A. Isidori, Nonlinear Control Systems II. London, U.K.: SpringerVerlag, 1999.

[7] Z. P. Jiang, A. Teel, and L. Praly, "Small-Gain theorem for ISS systems and applications," Math. Control, Signals, Syst., vol. 7, pp. 95-120, 1994.

[8] Z. P. Jiang, I. M. Y. Mareels, and Y. Wang, "A Lyapunov formulation of nonlinear small-gain theorem for interconnected systems," Automatica, vol. 32, pp. 1211-1215, 1996.

[9] Z. P. Jiang and I. M. Y. Mareels, "A small-gain control method for nonlinear cascaded systems with dynamic uncertainties," IEEE Trans. Automat. Contr., vol. 42, pp. 292-308, Feb. 1997.

[10] I. Karafyllis and J. Tsinias, "Global stabilization and asymptotic tracking for a class of nonlinear systems by means of time-varying feedback," Int. J. Robust Nonlinear Control, vol. 13, no. 6, pp. 559-588, 2003.

[11] — - "A converse Lyapunov theorem for nonuniform in time global asymptotic stability and its application to feedback stabilization," SIAM J. Control Optim., vol. 42, no. 3, pp. 936-965, 2003.

[12] - "Non-uniform in time stabilization for linear systems and tracking control for nonholonomic systems in chained form," Int. J. Control, vol. 76, no. 15, pp. 1536-1546, 2003.

[13] I. Karafyllis, "Non-uniform stabilization of control systems," IMA $J$. Math. Control Inform., vol. 19, no. 4, pp. 419-444, 2002.

[14] H. K. Khalil, Nonlinear Systems, 2nd ed. Upper Saddle River, NJ: Prentice-Hall, 1996.

[15] M. Krichman, E. D. Sontag, and Y. Wang, "Input-output-to-state stability," SIAM J. Control Optim., vol. 39, pp. 1874-1928, 1999.

[16] M.-C. Laiou and A. Astolfi, "Discontinuous control of high-order generalized chained systems," Syst. Control Lett., vol. 37, pp. 309-322, 1999.

[17] Y. Lin, E. D. Sontag, and Y. Wang, "A smooth converse Lyapunov theorem for robust stability," SIAM J. Control Optim., vol. 34, pp. 124-160, 1996.

[18] E. Panteley and A. Loria, "On global uniform asymptotic stability of nonlinear time-varying systems in cascade," Syst. Control Lett., vol. 33, no. 2, pp. 131-138, 1998.

[19] E. D. Sontag, "A universal construction of Artstein's theorem on nonlinear stabilization," Syst. Control Lett., vol. 13, pp. 117-123, 1989.

[20] — "Smooth stabilization implies coprime factorization," IEEE Trans. Automat. Contr., vol. 34, pp. 435-443, Mar. 1989.
[21] _ "Remarks on stabilization and input-to-State stability," in Proc. IEEE Conf. Decision Control, Tampa, FL, Dec. 1989, pp. 1376-1378.

[22] E. D. Sontag and Y. Wang, "On characterizations of the input-to-state stability property," Syst. Control Lett., vol. 24, pp. 351-359, 1995.

[23] — - "New characterizations of input-to-state stability," IEEE Trans. Automat. Contr., vol. 41, pp. 1283-1294, Sept. 1996.

[24] E. D. Sontag, "Comments on integral variants of ISS," Syst. Control Lett., vol. 34, pp. 93-100, 1998.

[25] E. D. Sontag and B. Ingalls, "A small-gain theorem with applications to input/output systems, incremental stability, detectability, and interconnections," J. Frank. Inst., vol. 339, pp. 211-229, 2002.

[26] A. Teel, "A nonlinear small gain theorem for the analysis of control systems with saturations," IEEE Trans. Automat. Contr., vol. 41, pp. 1256-1270, Sept. 1996.

[27] J. Tsinias, "Sufficient Lyapunov-like conditions for stabilization," Math. Control. Signals Syst., vol. 2, pp. 343-357, 1989.

[28] — - "Versions of Sontag's input to state stability condition and output feedback stabilization," J. Math. Syst. Estimat. Control, vol. 6, no. 1, pp. 113-126, 1996.

[29] - "Input to state stability properties of nonlinear systems and applications to bounded feedback stabilization using saturation," ESAIM Control, Optim. Calc. Var., vol. 2, pp. 57-85, 1997.

[30] J. Tsinias and I. Karafyllis, "ISS property for time-varying systems and application to partial-static feedback stabilization and asymptotic tracking," IEEE Trans. Automat. Contr., vol. 44, pp. 2179-2185, Dec. 1999.

[31] J. Tsinias, "Backstepping design for time-varying nonlinear systems with unknown parameters," Syst. Control Lett., vol. 39, no. 4, pp. 219-227, 2000.

[32] _ _ "A converse Lyapunov theorem for nonuniform in time, global exponential robust stability," Syst. Control Lett., vol. 44, no. 5, pp. 373-384, 2001

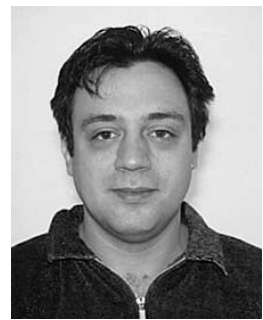

Iasson Karafyllis was born in Athens, Greece, in 1971. He received the B.S. degree in chemical engineering from the National Technical University of Athens (NTUA), Athens, Greece, in 1994, the M.Sc. degree in process integration from the University of Manchester Institute of Science and Technology, Manchester, U.K., in 1995, the M.Sc. degree in mathematics from the University of Minnesota, Minneapolis, in 1997, and the Ph.D. degree in mathematics from NTUA in 2003.

$\mathrm{He}$ is currently a Lecturer in the Department of Economics at the University of Athens. His research interests include mathematical control theory, stability theory and feedback stabilization problems for discrete-time, and continuous-time deterministic systems.

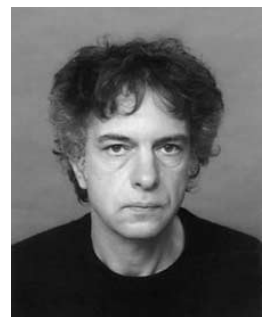

Iannis Tsinias received the B.S. degree in physics and the Ph.D. degree in systems science from the University of Athens, Athens, Greece, in 1978 and 1983, respectively.

He is currently a Professor in the Department of Mathematics, at the National Technical University of Athens, Athens, Greece. He was an Associate Editor for Systems and Control Letters and ESAIM-COCV, and he is currently a Member of the Editorial Board of the European Journal of Control. His research interests lie in nonlinear control theory. 